\title{
NOTE TECHNIQUE \\ LA SITUATION DES ÉCREVISSES EN FRANCE RÉSULTATS DES ENQUÊTES NATIONALES RÉALISÉES ENTRE 1977 ET 2006 PAR LE CONSEIL SUPÉRIEUR DE LA PÊCHE
}

\author{
COLLAS M., JULIEN C., MONNIER D.
}

Délégation régionale de l'Office national de l'eau et des milieux aquatiques de Metz - 23 rue des Garennes 57155 Marly

Courriel : marc.collas@onema.fr

Reçu le 20 mars 2007

Received March 20, 2007

Accepté le 11 septembre 2007

Accepted September 11, 2007

\section{RÉSUMÉ}

Depuis la fin du XIX ${ }^{\circ}$ siècle, la distribution des écrevisses en France a connu de profonds bouleversements, et l'introduction d'écrevisses allochtones en provenance essentiellement du continent nord-américain, a irrémédiablement modifié le paysage astacologique français et européen.

Les quatre enquêtes réalisées en France par le Conseil Supérieur de la Pêche depuis 1977, ont permis de suivre l'évolution des différentes espèces d'écrevisses recensées sur le territoire national, mettant à chaque fois en évidence la forte expansion des espèces exotiques et le recul des espèces natives.

L'enquête de 2006 n'échappe pas à cette tendance et la situation de nos trois espèces d'écrevisses doit désormais être considérée comme alarmante.

Deux de ces espèces se caractérisent par un statut de conservation proche de l'extinction (Austropotamobius torrentium et Astacus astacus) alors que la troisième, Austropotamobius pallipes est « réfugiée " sur les secteurs apicaux des bassins versants, confinée sur des zones soumises aux premières manifestations du changement climatique et isolées par les espèces exotiques qui progressent de plus en plus vers l'amont.

En effet, l'écrevisse du Pacifique (Pacifastacus leniusculus), mais aussi l'écrevisse rouge de Louisiane (Procamabrus clarkil), sont les espèces qui montrent la plus forte expansion géographique durant la période 2001-2006. Partout elles apparaissent comme des concurrents redoutables, plus agressifs, résistants aux pathologies, capables de coloniser des habitats variés....

Ces deux espèces en particulier étendent leur aire de répartition et colonisent chaque année de nouveaux départements, de nouveaux cours d'eau, éliminant par là même, les espèces autochtones.

L'expansion des écrevisses introduites, l'arrivée récente en France d'une nouvelle espèce (Orconectes juvenilis) et les modifications de la Loi autorisant dorénavant le transport à l'état vivant des "espèces susceptibles de provoquer des déséquilibres biologiques" pourraient sceller définitivement le sort des écrevisses endémiques, si aucune mesure n'est prise rapidement par les autorités, dans le cadre d'un plan d'action national « écrevisse ».

Mots-clés: écrevisses, répartition géographique, variation temporelle, gestion, conservation, France. 


\section{TECHNICAL NOTE \\ SITUATION OF THE CRAYFISH IN FRANCE \\ RESULTS OF THE NATIONAL SURVEYS PERFORMED BETWEEN 1977 AND 2006 BY THE CONSEIL SUPERIEUR DE LA PECHE (CSP)}

\section{ABSTRACT}

Since the end of the XIX ${ }^{\text {th }}$ century, the distribution of crayfish in France has gone through deep changes, and the introduction of allochtonous crayfish, essentially from the North American continent, has forever changed the French and European crayfish landscape.

The four surveys organised in France by the Conseil supérieur de la pêche since 1977 allow following the evolution of the different species of crayfish present on the national territory, showing each time the large expansion of the exotic species and the recess of the native species.

The 2006 survey shows the same tendency and the situation of our three native species must now be considered as alarming.

Two of these species, Austropotamobius torrentium and Astacus astacus, are close to extinction, and the third one, Austropotamobius pallipes, can now be found on the upmost parts of the watersheds, confined in areas where the first signs of climatic changes can be seen, and under the pressure of the exotic species which are still making their way further up.

Pacifastacus leniusculus and Procamabrus clarkii are showing the strongest geographical expansion during the 2001-2006 period. Everywhere they appear to be very strong competitors, more aggressive, resistant to pathologies, able to colonize varied environments. They are indeed colonizing new departments, new watersheds, and thus eliminating native species.

Keywords : crayfish, geographical distribution, temporal variation, management, conservation, France.

\section{INTRODUCTION}

Les enquêtes nationales sur la répartition des écrevisses en France menées en 1977, 1988, 1995 et 2001 auprès des brigades du Conseil Supérieur de la Pêche (CSP) ont montré que l'évolution des populations d'écrevisses était très rapide, se traduisant notamment par deux tendances bien identifiées: recul et raréfaction des écrevisses endémiques, progression constante des écrevisses exotiques introduites (CHANGEUX, 2003).

Cette situation a suscité la mise en place d'une veille écologique spécifique à l'échelon national, initiée par le CSP, et encadrée au niveau régional par les différentes délégations régionales de l'établissement. L'enquête menée en 2006 porte donc sur la période 2001-2006. Elle doit en effet permettre de vérifier ces tendances et d'acquérir de nouvelles connaissances sur la situation spécifique des différentes espèces et sur leur répartition géographique.

Jusqu'en 2001, sept espèces d'écrevisses étaient identifiées en France :

- trois espèces autochtones, l'écrevisse à pieds blancs (Austropotamobius pallipes), espèce la plus largement répandue au niveau national, l'écrevisse à pattes rouges (Astacus astacus) dont la répartition naturelle semble couvrir l'Est du pays et plus particulièrement les bassins de la Moselle et de la Meuse (LAURENT et SUSCILLON, 
1962), et enfin l'écrevisse des torrents (Austropotamobius torrentium), initialement signalée en Alsace (Lereboullet, 1858), longtemps considérée disparue et redécouverte dans le Nord-Est du pays dans deux départements (MACHINO, 1995 ; MACHINO, 1996 ; COLLAS, 1996, FRANCKHAUSER et MACHINO, 2001, HORNIER et al., 2002).

- quatre espèces introduites, avec par ordre d'apparition, l'écrevisse américaine (Orconectes limosus), originaire de la côte Est des Etats-Unis, l'écrevisse à pattes grêles (Astacus leptodactylus), originaire d'Asie mineure et centrale, l'écrevisse du Pacifique ou écrevisse signal (Pacifastacus leniusculus) provenant de la côte Ouest des Etats-Unis et l'écrevisse rouge de Louisiane (Procambarus clarkii) quant à elle issue des bayous du Mississippi.

Presque un siècle après l'introduction de la première écrevisse exotique en France, une nouvelle espèce Orconectes juvenilis (COLLAS et TAYLOR, à paraître) fait son apparition dans la faune astacicole française, découverte en 2005 dans le département du Doubs ; elle est également originaire d'Amérique du Nord.

Son apparition pourrait en entraîner d'autres dans un futur proche, la parution récente d'un atlas sur la répartition des différentes espèces en Europe (SOUTY-GROSSET et al., 2006) signale la présence de plusieurs écrevisses exotiques dans différentes régions d'Europe (Orconectes virilis, Orconectes immunis, Procambarus sp., et Cherax destructor, originaire d'Australie).

Ce document présente les résultats de l'enquête 2006 et synthétise sous forme de carte de répartition, l'ensemble des données pour les huit espèces recensées dans les différents milieux naturels aquatiques du territoire national.

Enfin, il aborde sommairement les menaces qui pèsent sur les dernières populations d'écrevisses indigènes.

\section{METHODE}

La méthodologie utilisée lors des enquêtes précédentes a été reprise en 2006. Un questionnaire d'enquête "express écrevisses ", semblable à celui employé en 2001, a été diffusé par la Direction Générale du CSP à l'ensemble des délégations régionales au cours du mois de septembre. Dans chacune des délégations régionales un correspondant " écrevisses " était chargé de le diffuser auprès des brigades départementales puis de collecter et de centraliser les données avant de les transmettre au correspondant national pour exploitation.

Un premier travail de vérification a été réalisé au niveau régional, cette étape a donné lieu à de nombreux échanges entre le correspondant régional et les brigades départementales. Ces échanges se sont poursuivis entre les correspondants régionaux et le correspondant national, les cartes de répartition ont ainsi fait l'objet d'une validation dans chaque délégation régionale.

Par rapport aux enquêtes précédentes, on peut cependant observer plusieurs évolutions du questionnaire. Ainsi, et afin de préciser le statut biologique de chacune des espèces, outre la notion de présence/absence, il était demandé de préciser le nombre de sites pour chaque espèce mentionnée dans le département. La valeur devait permettre d'apprécier l'abondance de chaque espèce au niveau départemental.

D'autre part, il paraissait intéressant de recueillir des données sur les mortalités d'écrevisses en France; une rubrique supplémentaire a donc été ajoutée au questionnaire.

Le questionnaire à remplir par chaque brigade (voir copie en annexe 1), porte sur l'ensemble du département sans faire intervenir de localisation plus détaillée. Chacune 
des cartes, présentées dans ce document, résume les informations concernant aussi bien les eaux libres (cours d'eau, lacs et retenues de $1^{\circ}$ et $2^{\circ}$ catégorie) que les eaux closes et les piscicultures. L'évolution à court terme a été appréciée en distinguant 3 classes: (1), disparition de population, (2), maintien des populations, (3), apparition de populations.

Pour suivre l'évolution à long terme de la répartition, espèce par espèce, des cartes semblables ont été réalisées à partir des enquêtes précédentes (1977, 1990, 1995 et 2001). Les protocoles adoptés au cours des différentes enquêtes ont été suffisamment similaires pour qu'elles soient comparables entre elles. Ces indications sont utilisées dans la suite pour parler d'apparition ou de disparition, de diminution ou d'extension. Toutefois, comme c'est généralement le cas pour les informations de répartition, si la présence d'une espèce (représentée en grisé) est une donnée avérée, l'absence (représentée en blanc) est une information moins définitive car il n'est pas possible de mettre en œuvre une prospection intégrale des milieux aquatiques.

\section{Difficultés rencontrées}

Etablir une carte de répartition nationale d'une espèce est un projet ambitieux qui se trouve confronté à différentes difficultés. Le niveau de connaissance général est très variable d'un département à l'autre, mais aussi d'une espèce à l'autre. L'information sur la localisation des espèces est souvent dispersée, aucune base de données n'existant actuellement au niveau national. La réalisation de l'enquête 2006 n'a pas échappé à ces difficultés et l'on peut globalement distinguer deux situations qui sont apparues lors de l'exploitation des réponses.

\section{Ecrevisses endémiques}

Les réponses au questionnaire font ressortir un niveau de connaissance que l'on peut qualifier de bon et qui se manifeste notamment par l'intérêt des agents pour ces espèces "vulnérables", selon le classement établi par l'Union Internationale pour la Conservation de la Nature (UICN).

Cette connaissance globale de la situation départementale est généralement renforcée par des études ponctuelles destinées à proposer des mesures de gestion et de conservation (Natura 2000) ou par la réalisation d'atlas départementaux de répartition. Ces documents, souvent réalisés avec différents partenaires (Agence de l'Eau, Parcs Naturels Régionaux, Fédérations de pêche...) permettent souvent de mettre par écrit les connaissances historiques des agents de terrain.

Pour les trois espèces endémiques, les cartes de répartition publiées dans ce document font donc apparaître le nombre de sites actuellement connus dans chaque département.

\section{Ecrevisses introduites}

Plusieurs cas doivent être distingués. D'une manière générale la connaissance de ces espèces dans les eaux libres (au sens de la réglementation) peut également être qualifiée de bonne. Les études citées plus haut permettent également de suivre, en parallèle, l'évolution des populations de ces espèces.

Pour ces espèces et à l'exception d'Orconectes juvenilis, les cartes publiées ne mentionnent pas le nombre de sites connus dans les départements, car les informations transmises sont généralement trop lacunaires ou imprécises sur les plans d'eau du domaine privé notamment.

\section{Le cas des plans d'eau}

La situation se complique davantage lorsque l'enquête aborde la question des plans d'eau, plus particulièrement celle relative aux eaux closes, piscicultures et plans 
d'eau privés. Ces ouvrages, constituent un vecteur de dispersion des espèces introduites non négligeable, pour lesquels il est souvent difficile d'obtenir des renseignements fiables (aussi bien au niveau administratif qu'écologique). Dans certains départements leur nombre est tellement important (plusieurs milliers) qu'il est illusoire mais surtout très difficile, de vouloir connaître quelles espèces les peuplent. Cependant, s'agissant des plans d'eau du domaine public, où les agents sont amenés à intervenir régulièrement, la connaissance est satisfaisante.

\section{La notion de sites}

Cette notion, suite aux modifications enregistrées dans la répartition des écrevisses en France, s'applique de façon satisfaisante aux espèces endémiques qui occupent généralement des linéaires de cours d'eau peu importants. En effet, les écrevisses autochtones sont aujourd'hui localisées sur des tronçons de cours d'eau facilement identifiables, à la faveur notamment des prospections nocturnes. Ainsi, plusieurs sites peuvent être observés sur un même cours d'eau ou un bassin versant. La taille d'un site n'est donc pas un critère homogène.

Elle s'applique toutefois plus difficilement aux écrevisses exotiques qui sont capables de coloniser des linéaires entiers de cours d'eau. L'exemple de l'écrevisse américaine est le cas le plus fréquent, l'espèce est en effet censée occuper tous les grands axes fluviaux sur des linéaires importants. Dans ce cas, le nombre de sites sous-estime le niveau réel des populations au niveau départemental et donc au niveau national.

D'autre part certaines d'entre elles (Procambarus clarkii) sont observées dans des milieux très variés (marais, étangs littoraux...), où la surface colonisée est parfois très importante.

\section{RÉSULTATS}

Parmi les indications qui ressortent de l'exploitation des questionnaires, plusieurs éléments semblent ressortir. Les brigades du Conseil Supérieur de la Pêche, montrent un intérêt réel au suivi des populations d'écrevisses. Cette tendance se vérifie notamment au travers des nombreuses études réalisées au niveau départemental, à la mise en œuvre d'outils informatiques destinés à géo-localiser les sites (base EXCEL, ACCESS...).

D'autre part, il ressort de l'enquête réalisée en 2006 un intérêt manifeste pour deux espèces en particulier, l'écrevisse à pieds blancs (APP) et l'écrevisse du Pacifique (PFL). La première fait l'objet de recherches actives pour son intérêt en tant qu'espèce patrimoniale, la seconde est souvent découverte au profit d'études ponctuelles ou signalée par les usagers des milieux aquatiques. Ces deux espèces, bien représentées au niveau national, occupent des habitats similaires, et la recherche de sites à écrevisses à pieds blancs aboutit parfois à la découverte d'une population de P. lenisuculus ou d'Orconectes limosus.

Les autres espèces endémiques ( $A$. astacus, $A$. torrentium), dont la répartition est plus retreinte au niveau national sont également bien suivies au niveau local. Pour les trois espèces endémiques, il a donc été possible de dresser des cartes de répartition indiquant le nombre de sites connus par département.

\section{Situation des espèces autochtones}

Les causes du déclin des espèces autochtones sont multiples, on peut citer l'apparition en Europe vers 1860 d'une pathologie (l'Aphanomycose ou peste des écrevisses) qui a décimé les populations à travers toute l'Europe. Cette pathologie serait apparue en France vers 1875 et aurait décimé les populations sur l'ensemble du territoire national en un peu moins d'une décennie. Partout, des mortalités massives ont été observées sans qu'aucun remède ne puisse être apporté. Cette pathologie continue de sévir aujourd'hui, comme en attestent des observations récentes. 
La dégradation des milieux naturels liée aux aménagements (travaux dans le lit mineur, aménagement des bassins versants, création d'étangs...), l'altération de la qualité de l'eau (pollution) sont également à l'origine de cette tendance.

En effet, les écosystèmes aquatiques intègrent toutes les conséquences des activités humaines, ils subissent de profondes perturbations et parmi les principales qui agissent sur l'habitat de l'écrevisse, on peut citer :

- La pollution des cours d'eau : l'écrevisse est très sensible à la qualité de l'eau, les pollutions ponctuelles ou chroniques (industrielles, domestiques ou agricoles) fragilisent ou détruisent les populations ;

- La destruction de l'habitat: la modification de l'habitat suite à la réalisation de travaux à but hydraulique (curage, recalibrage, rectification) entraîne une uniformisation et une banalisation de tous les habitats nécessaires aux différents stades de développement de l'écrevisse. D'autre part, l'évolution des pratiques agricoles a entraîné de profondes modifications à l'échelle des bassins versants. Elles se sont traduites par une évolution de l'occupation des sols, disparition des prairies au profit de monocultures intensives nécessitant des traitements par pesticides, des apports d'engrais....

Cette évolution s'est généralement accompagnée de travaux d'aménagement des cours d'eau qui ont profondément et durablement modifié la structure physique de ces milieux. Réalisés à des fins hydrauliques, ces travaux ont banalisé les habitats de nombreuses rivières de plaine, détruisant par là même l'un des principaux habitats de l'écrevisse à pattes rouges.

Enfin, l'introduction d'espèces exotiques est également une cause de dégradation et de disparition des populations endémiques. Aujourd'hui, les dernières populations d'écrevisses européennes sont menacées par ces espèces qui présentent des traits biologiques plus favorables (stratégie démographique, résistance aux pathologies...).

Parmi les cinq espèces d'écrevisses introduites, quatre espèces sont issues d'Amérique du Nord: Toutes ces espèces du Nouveau monde sont porteuses saines du champignon (Aphanomyces astacl) responsable de la peste des écrevisses ou aphanomycose, et rentrent en compétition avec les espèces autochtones. Ainsi, en terme de compétition inter-spécifique, l'écrevisse du Pacifique doit actuellement être considérée comme la principale concurrente de l'écrevisse à pieds blancs. Les deux espèces, qui occupent des habitats similaires, entrent aujourd'hui en compétition sur de nombreux bassins versants, avec dans la majorité des cas observés, un avantage pour l'espèce introduite.

D'autre part les secteurs abandonnés par l'écrevisse à pattes rouges ou l'écrevisse à pieds blancs ont été pour la plupart colonisés par des espèces exotiques moins exigeantes sans aucune possibilité de reconquête.

\section{Statut réglementaire}

Les espèces endémiques font l'objet de différents niveaux de protection par des textes nationaux ou internationaux (FIERS et al., 1997).

\section{L'écrevisse à pieds blancs - Austropotamobius pallipes (Lereboullet, 1858) - code APP}

Considérée comme un indicateur de milieu de bonne qualité, l'écrevisse à pieds blancs fait l'objet d'une attention et d'un suivi particulier de la part des brigades départementales; des efforts spécifiques de prospection ont été réalisés dans un grand nombre de départements. 
Autrefois largement répandue sur l'ensemble du réseau hydrographique, l'espèce est aujourd'hui essentiellement localisée sur les zones amont des hydrosystèmes, où les populations sont isolées, sans continuum écologique.

Le preferendum écologique de cette espèce exigeante, bien que centré sur la zone à truite décrite par HUET (1949), est relativement large avec des extensions dans la zone à barbeau. Selon la typologie établie par VERNEAUX (1973), la gamme typologique de l'écrevisse à pieds blancs s'étendrait au moins du B2 au B7 (TELEOS, 2004) avec un optimum typologique pour des niveaux compris entre B3 et B5.

\section{Situation en 2006 (Figure 1)}

Ainsi, l'enquête permet de recenser Austropotamobius pallipes dans 76 départements sur un total de 2249 sites. Les plus fortes concentrations sont observées sur le domaine continental, plus précisément dans les régions Bourgogne, Franche-Comté Rhône-Alpes, Auvergne, mais aussi sur les domaines méditerranéen (Languedoc-Roussillon) et alpin. II est important toutefois de pondérer ces concentrations par les efforts de prospection qui peuvent modifier le statut de l'espèce. En Bourgogne où 1400 prospections ont été réalisées depuis 1999, une relation a été mise en évidence entre le nombre de sites à écrevisses pieds blancs et le nombre total de sites prospectés (LERAT et al., 2006).

L'écrevisse à pieds blancs demeure l'espèce endémique la mieux représentée en France, même si elle est absente ou n'est plus signalée dans 20 départements. Pour huit d'entre eux l'espèce avait pourtant été mentionnée par LAURENT et SUSCILLON (1962) : Aube, Charente, Landes, Nord, Pas-de-Calais, Bas-Rhin, Seine-et-marne, Somme. Dans ces départements et malgré les efforts de prospection, elle peut déjà être considérée comme disparue ou au bord de l'extinction. En Charente-Maritime et malgré deux années de prospection, l'espèce est considérée disparue depuis 2000.

Ses populations peuvent être considérées comme « reliques » dans vingt autres, où le nombre de sites abritant des écrevisses est inférieur à dix. Dans l'Hérault, la brigade observe l'apparition de Pacifastacus leniusculus sur le dernier secteur où les pieds blancs présentent une population intéressante; dans l'Indre comme dans plusieurs autres départements elle est présente sous forme "relictuelle ", la Lozère mentionne que les populations diminuent ou disparaissent totalement sur des axes importants. Dans les observations faites par les brigades, les mentions " régression généralisée des populations, effondrement des effectifs, disparition de populations... " sont souvent utilisées par les brigades pour qualifier l'état de conservation de l'écrevisse à pieds blancs.

Ces observations sont confirmées par un nombre élevé de cas de mortalités. Ainsi, l'écrevisse à pieds blancs représente à elle seule $89 \%$ des cas signalés en France pendant la période 2001/2006, 41 départements en font état avec des origines diverses : événements climatiques observés depuis 2003 et épizooties diverses (Aphanomycose et Thélohaniose). Les brigades ne précisent pas si les mortalités sont totales et si des possibilités de reconquête existent.

En terme de tendance, $20 \%$ des brigades signalent la disparition de populations, $45 \%$ signalent le maintien des populations et $10 \%$ signalent de « nouvelles populations ", dont la découverte est vraisemblablement à mettre en relation avec l'effort de prospection en augmentation pour cette espèce.

Par rapport à l'enquête de 2001, on observe que $A$. pallipes est signalée dans cinq nouveaux départements : l'Oise ( 3 sites), l'Aisne ( 3 sites), la Meurthe-et-Moselle (1 site), l'Essonne ( 1 site) et la Vendée (1 site). Dans ces départements, les prospections nocturnes réalisées dans le but de rechercher cette espèce ont permis de la mettre en évidence alors que dans certains cas (Meurthe-et-Moselle, Oise, Essonne), elle n'avait jamais été mentionnée auparavant (CHANGEUX, 2003). 
On constate également que l'espèce n'est plus mentionnée dans deux départements, le Pas de Calais (uniquement mentionnée en 2001) et le département de la Creuse, où selon la brigade départementale l'ensemble des sites connus a été déserté suite aux mortalités massives observées au cours de l'année 2001 et dont l'origine n'a pu être déterminée. Pour ce département on observe pour la période concernée un développement très important de l'écrevisse du Pacifique.

L'enquête montre également qu'Austropotamobius pallipes est une espèce surtout présente dans les cours d'eau, bien qu'elle soit parfois mentionnée en plans d'eau (10 cas). Elle occupe surtout les cours d'eau à vocation salmonicole (2017 sites), et n'est que peu signalée sur les cours d'eau de seconde catégorie piscicole (222 sites).

L'aire de répartition de cette espèce couvre les quatre domaines biogéographiques (Figure 1).

- l'espèce semble moins bien représentée sur le domaine atlantique, elle n'est pas mentionnée au Centre et au Nord (région parisienne et Nord de la France), mais aussi sur une grande partie de la façade Ouest ;

- sur le domaine méditerranéen, l'espèce est présente dans tous les départements, avec des niveaux de population variables. C'est dans les Bouches du Rhône que sa situation semble la plus délicate

- sur le domaine continental, l'espèce est présente dans l'ensemble des départements à l'exception toutefois des départements de l'Aube et du Bas-Rhin, où l'espèce était historiquement présente (BALDNER, 1888 ; LAURENT et SUSCILLON, 1962 ; ANDRE, 1960) mais où elle n'est plus observée (disparition probable) malgré des recherches actives localement. En outre l'espèce n'est plus mentionnée dans le département de la Creuse (mortalités massives en 2001) ; dans le département voisin de la Haute-Vienne, une seule population qualifiée de « relictuelle » est signalée.

- enfin sur le domaine alpin, l'espèce est présente en Haute-Savoie, en Savoie, en Isère et dans les Hautes-Alpes. Les populations recensées voient leur aire de répartition colonisée par l'écrevisse du Pacifique, bien implantée dans le Lac Léman et dont l'expansion semble très rapide.

Sur les 76 départements où l'espèce est recensée, la pêche est autorisée dans 34 départements et interdite dans 42 départements. Là où l'exploitation est autorisée l'espèce fait généralement l'objet d'une pêche par les amateurs que l'on peut qualifier d'active, la période de 10 jours d'ouverture prévue par la loi est parfois raccourcie afin de limiter les prélèvements sur l'espèce.

\section{L'écrevisse à pattes rouges - Astacus astacus (Linnaeus, 1758) - Code ASA}

L'écrevisse à pattes rouges est une espèce d'Europe centrale qui touche en France les limites occidentales de son aire de répartition. Les différentes enquêtes montrent que l'espèce se rencontre surtout dans le Nord-Est (régions Alsace, Lorraine, ChampagneArdenne et Bourgogne).

Au niveau national, il semble que l'espèce ait toujours fait l'objet de nombreux essais de transplantation, d'abord en vue de sa réintroduction suite aux épidémies de peste et ensuite à des fins de production extensive. Ces expérimentations ont connu des succès divers. Cette espèce affectionne les eaux calmes des rivières à cours lent, et des plans d'eau. Au niveau typologique, on la rencontre généralement dans les petites rivières de plaine. La bibliographie et les mentions historiques signalent que l'espèce fréquentait les grandes rivières (zone à brème) comme la Moselle et ses affluents aux alentours de Metz (PEUPION, 1898), ou la Meuse à Verdun, I'Ill aux portes de Strasbourg (BALDNER, 1888). 

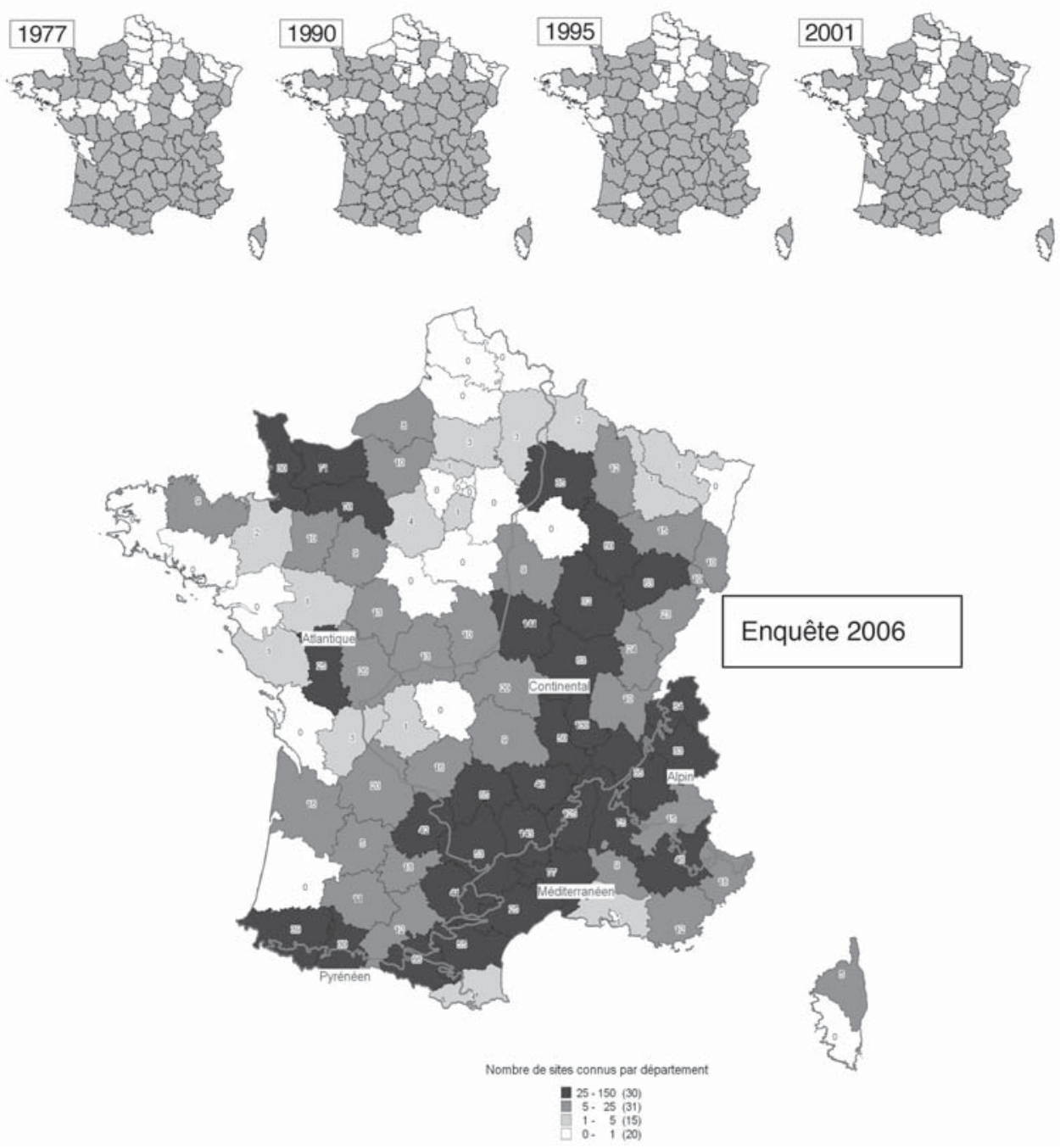

\section{Figure 1}

Evolution de la répartition de l'écrevisse à pieds blancs (Austropotamobius pallipes). Pour les cartes des années 1977 à 2001, le signalement de l'espèce est figuré par un grisé. Pour la carte 2006, l'intensité des bleus reflète l'abondance de l'espèce à l'intérieur de chaque département, conformément à la légende.

\section{Figure 1}

Evolution of the repartition of A. pallipes. On the 1977-2001 maps, the departments with observation of the species is indicated in grey. On the 2006 map, the intensity of the blue is related to the number of observations made in each department.

Dans son ouvrage, PEUPION (1898) mentionne que l'écrevisse à pattes rouges "se rencontre d'abord dans les cours d'eau où vivent les poissons tels que carpe, tanche, chevesne, poissons blancs divers et enfin perche et brochet ", il précise également " on les trouvait dans toutes les rivières en Lorraine et en Alsace-Lorraine... la Meuse sur tout son cours français et belge... la Moselle dont le cours en Prusse était plus productif que le cours français... La Meurthe, la Sarre, l'Ill et l'Aube ». 
Ses populations ont été décimées par l'apparition de l'Aphanomycose (peste des écrevisses) à la fin du XIX ${ }^{\circ}$ siècle. PEUPION (1898) indique « il a fallu tout au plus une année pour que la destruction fut complète dans tout le cours de la Moselle, de même dans celui de la Meuse, la ruine des cours d'eau lorrains et belges date de l'année 1878 ". DE DROUIN DE BOUVILLE (1906) précise que "les premières manifestations eurent lieu en 1876, en différents point d'une assez vaste superficie, celle occupée par les départements de la Meuse, de Meurthe-et-Moselle, des Vosges, de la Haute-Saône, du Jura, de la Côte d'Or, de la Nièvre, de l'Yonne et de l'Aube. Le mal paraît avoir pris naissance en Lorraine ". II indique également " $A$ la fin de l'année 1885, la peste avait exercé son action dévastatrice sur presque toute la surface du territoire français ».

En une décennie, les peuplements ont été décimés. De nombreuses opérations de repeuplement ont dès lors été entreprises dans ces régions, ainsi dans le département de Meurthe-et-Moselle, 75000 écrevisses en provenance de Silésie et de Pologne ont été déversées dans les cours d'eau du département à partir de 1881 grâce à des crédits votés par les Conseils Généraux (DE DROUIN DE BOUVILLE, 1901). Des tentatives identiques ont eu lieu dans le département de la Moselle et des Vosges (COLLAS, données non publiées).

\section{Situation en 2006 (Figure 2)}

En 2006 l'espèce est mentionnée dans 21 départements (16 en 2001), sur 115 sites. 36 sont situés sur des cours d'eau de première catégorie piscicole et 8 sur la seconde catégorie piscicole, 71 sites correspondent à des plans d'eau (62\% des sites).

L'aire de répartition naturelle couvre la partie Nord-Est du domaine continental, c'est à dire l'Alsace (Bas-Rhin : 11 sites), la Lorraine, région qui regroupe à elle seule près de $50 \%$ des sites recensés, où l'espèce est encore observée dans les quatre départements, et c'est en Moselle qu'elle semble la plus abondante (36 sites localisés pour la majorité dans les Vosges du Nord).

L'espèce est également observée en Champagne-Ardenne dans les départements de la Haute-Marne ( 7 sites), où suite à son introduction dans des plans d'eau elle a également colonisé certains cours d'eau, dans la Marne (4 sites) et dans les Ardennes (1 site). Les inventaires réalisés dans ces départements ces dernières années ont permis de préciser le statut de cette espèce dans cette région et de mettre en évidence des populations méconnues (JULIEN et al. ; 2005).

La Bourgogne (Nièvre : 9 sites, Yonne : 2 sites en plans d'eau (BLATTER com.pers.)) et la Franche-Comté (Haute-Saône : 11 sites) semblent constituer la limite sud-ouest de répartition.

Globalement même si l'aire de répartition de cette espèce s'est étendue au niveau national, on constate qu'elle est principalement le fait de tentatives d'introductions à des fins d'élevage (astaciculture extensive).

Plus localement, et dans son aire de répartition naturelle, les populations continuent de régresser et de disparaître avec des mortalités massives sur certains sites sans identification précise de l'agent pathogène (BARAN, com.pers.). L'espèce réussit à se maintenir dans de petits plans d'eau privés, mais on peut la considérer comme disparue des rivières de plaine qui constituaient autrefois son habitat de prédilection et où elle était abondante.

L'espèce, qui a aujourd'hui déserté la quasi-totalité du réseau hydrographique subsiste essentiellement dans des petits plans d'eau forestiers, exempts de pollution, et où les espèces exotiques sont absentes. Elle colonise parfois l'émissaire des plans d'eau, mais son implantation est souvent limitée. L'espèce concurrence l'écrevisse des torrents, suite à son introduction dans des plans d'eau, d'où elle s'est échappée (COLLAS et al., 2005b). 

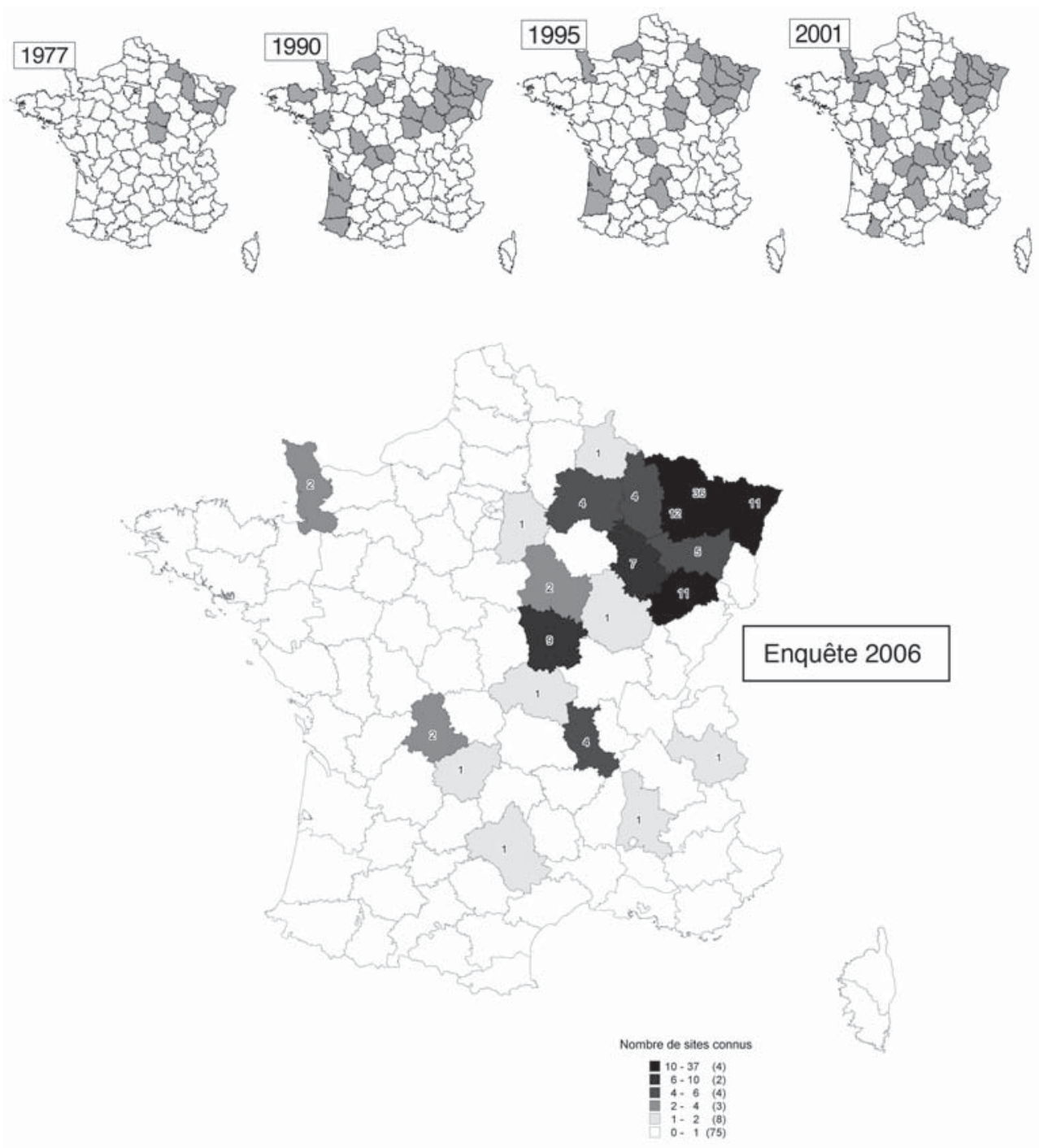

Figure 2

Evolution de la répartition de l'écrevisse à pattes rouges (Astacus astacus). Pour les cartes des années 1977 à 2001, le signalement de l'espèce est figuré par un grisé. Pour la carte 2006, l'intensité des bleus reflète l'abondance de l'espèce à l'intérieur de chaque département conformément à la légende.

\section{Figure 2}

Evolution of the geographical repartition of Astacus astacus. On the 1977-2001 maps, the departments where the species has been observed is shaded in grey. On the 2006 map, the intensity of the blue is related to the number of observations made in each department.

Par rapport à 2001, ASA est observée dans sept départements supplémentaires généralement sur un seul site et souvent suite à une opération de réintroduction (Allier, Côte d'Or, Drôme, Seine-et-Marne); seul le département de la Haute-Vienne compte 2 sites, alors que dans la Marne l'espèce est introduite dans des étangs conservatoires (GUIDOU, Com.pers.). Enfin, l'espèce est à nouveau signalée dans le département des Ardennes (déjà signalée en 1977 et 1995) sur un cours d'eau de première catégorie piscicole. 
Par contre, elle n'est plus mentionnée dans le Cantal et le Puy-de-Dôme.

La pêche de cette espèce est très limitée, en raison de sa disparition sur le réseau hydrographique. Elle est toutefois prélevée dans quelques plans d'eau privés pour alimenter la restauration locale. C'est le cas dans les Vosges du Nord, où un projet de développement d'étangs conservatoires est en cours de réalisation.

La situation de $A$. astacus doit donc être considérée comme alarmante. Actuellement, la survie de cette espèce est directement liée à son intérêt gastronomique et aux essais d'astaciculture extensive dont elle fait l'objet par des particuliers.

\section{Code APT \\ L'écrevisse des torrents - Austropotamobius torrentium (Schranck, 1803) -}

L'aire de répartition de l'écrevisse des torrents concerne principalement l'Europe centrale. En France l'espèce touche à la limite occidentale de son aire de répartition, le bassin de la Moselle constituerait la limite ouest de distribution.

L'espèce est uniquement observée sur le domaine continental. Elle avait été signalée pour la première fois en France, plus précisément en Alsace par LEREBOULLET (1858) sous le nom d'Astacus longicornis. L'espèce était également signalée en 1935 sur les rivières III et Bruche (ANDRÉ et LAMY, 1935 ), et selon les informations bibliographiques elle était abondante sur les marchés de Strasbourg. Cependant elle n'avait plus jamais été mentionnée dans les différents inventaires astacologiques.

Longtemps considérée comme disparue de la faune française, cette écrevisse est redécouverte, d'abord en Lorraine (MACHINO, 1995 ; MACHINO, 1996 ; COLLAS, 1996 ; FRANCKHAUSER et MACHINO, 2001) dans le département de la Moselle, qui est actuellement le seul département français où l'on peut observer les trois espèces d'écrevisses autochtones, puis en Alsace dans le département du Bas-Rhin (HORNIER et al., 2002). Dans cette période trois sites abritant l'écrevisse des torrents ont été recensés ; l'un d'eux a déjà disparu.

\section{Situation en 2006 (Figure 3)}

Actuellement l'espèce est observée sur deux sites correspondant à de petits cours d'eau situés en tête de bassin. Une étude réalisée en 2005 (COLLAS et al., 2005a) a permis de mettre en évidence que les deux dernières populations d'écrevisse des torrents étaient fragilisées par différentes perturbations et que le statut biologique de cette espèce en France devait être considéré comme critique.

Les deux populations d'écrevisse des torrents connues à ce jour en France sont localisées dans le périmètre du Parc Naturel Régional des Vosges du Nord. L'une d'elles est localisée à l'extrémité ouest du parc, dans le département de la Moselle, et l'autre à l'extrémité est, dans le département du Bas-Rhin. La population mosellane est située sur le bassin de la Moselle, tandis que dans le Bas-Rhin, la population est localisée sur le bassin du Rhin.

Actuellement l'habitat de l'écrevisse des torrents correspond à des zones refuges sur des secteurs apicaux où les activités anthropiques sont très peu développées, et qui, dans les deux cas observés, correspondent à la zone à truite amont. Ses espèces d'accompagnement sont la Lamproie de Planer (Lampettra planeri) le vairon (Phoxinus phoxinus) et la loche franche (Barbatula barbatula).

Historiquement, il semble que cette espèce faisait l'objet d'une répartition plus large et qu'elle occupait une gamme typologique plus étendue. Les perturbations subies par les parties intermédiaires des cours d'eau ont largement contribué à la disparition de l'espèce sur ces secteurs. 

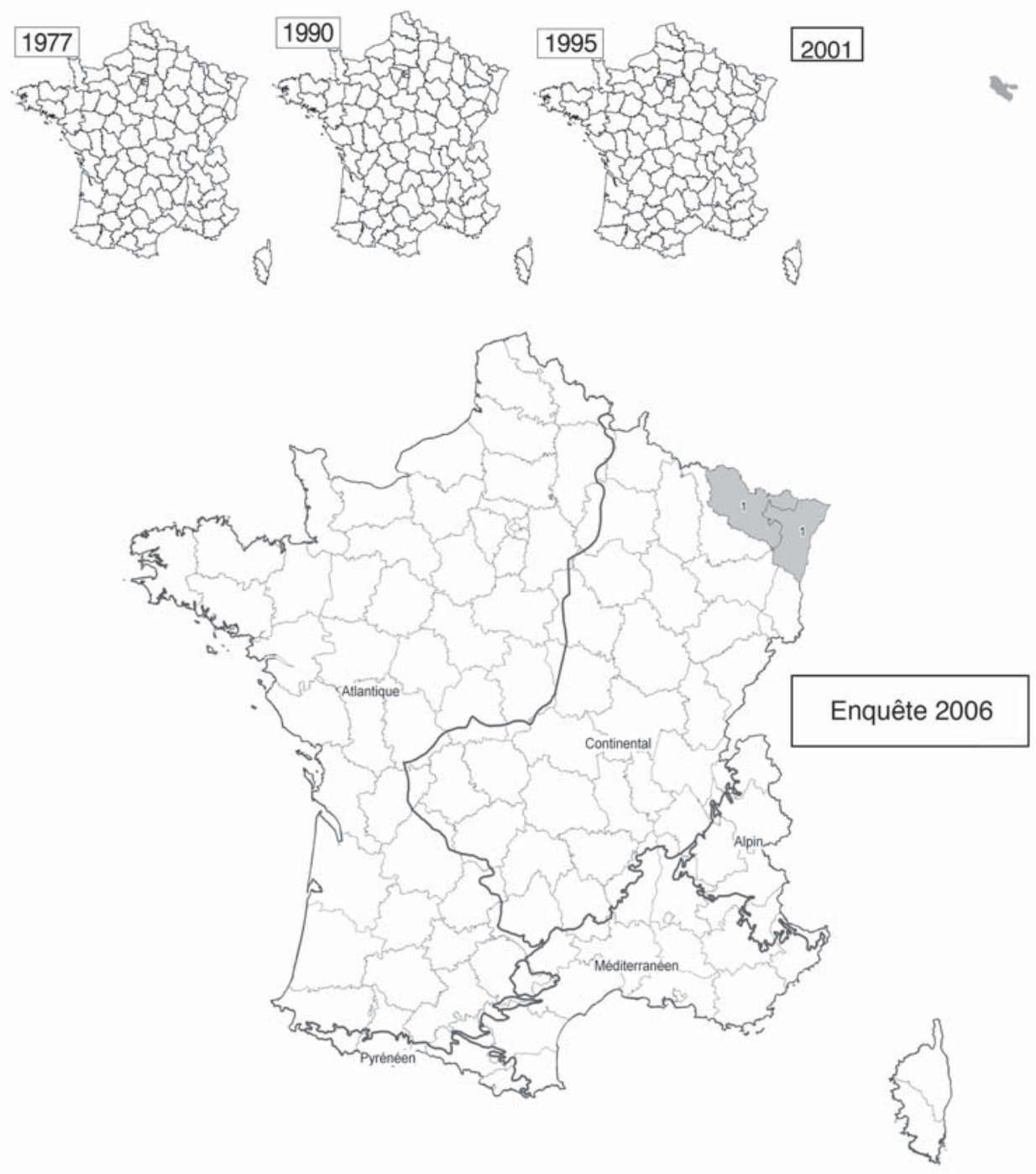

Figure 3

Evolution de la répartition de l'écrevisse des torrents (Austropotamobius torrentium). Le signalement de l'espèce est figuré par un grisé du département concerné, de 1977 à 2001, puis en 2006 par un aplat bleu.

Figure 3

Evolution of the geographical repartition of Austropotamobius torrentium. The departments where the species has been observed is shaded in grey.

En matière de pêche, le prélèvement de l'écrevisse des torrents est interdit en Moselle mais demeure autorisé par l'Arrêté préfectoral du Bas-Rhin.

L'enquête 2006 montre que le statut de cette espèce en France est préoccupant, selon les critères retenus par l'Union Internationale de Conservation de la Nature (KEITH et al., 1992), l'espèce est considérée comme "vulnérable ", c'est à dire qu'il s'agit d'une espèce dont les effectifs sont en forte régression du fait de facteurs extérieurs défavorables. Cependant l'espèce doit désormais être considérée en France "en danger » (espèces ayant déjà disparu d'une grande partie de leurs aires d'origines et dont les effectifs sont réduits à un seuil minimal critique. L'espèce est menacée de disparition si les causes 
responsables de leur situation actuelle continuent d'agin). Des mesures spécifiques de préservation et de protection doivent être mises en œuvre.

Malgré son inscription récente en 2000 sur la liste des espèces protégées, les mesures réglementaires (protection de l'espèce et de son habitat) semblent insuffisantes pour permettre à elles seules sa préservation.

\section{Situation des espèces exotiques introduites:}

L'apparition de la peste de l'écrevisse à la fin du XIXº siècle a encouragé l'introduction d'espèces exotiques résistantes à cette pathologie et destinées à compenser la disparition des écrevisses européennes.

C'est d'abord Orconectes limosus qui a gagné l'Europe par l'intermédiaire d'un pisciculteur allemand en 1890. En France des essais d'acclimatation sont initiés par RAVARET-WATTEL en 1896 près de Fécamp, selon LEGER (1924) deux espèces sont concernées (Cambarus affinis et Cambarus virilis). Cambarus affinis (nom ancien d'O. Limosus) est introduite par un amateur dans le Cher près de Saint-Florent de 1911 à 1913, 2000 individus importés d'Allemagne sont ainsi déversés dans le cours d'eau (BUFFAULT, 1925 ; ANDRE, 1938a et b). Cette introduction constitue le point de départ de la propagation de l'écrevisse américaine à travers la France.

L'origine de l'introduction de $A$. leptodactylus, espèce originaire d'Asie mineure et centrale (bassin du Danube) semble plus difficile à préciser. Selon LAURENT (2003), l'espèce aurait pu être acclimatée en France au tournant des $19^{\text {ème }}$ et $20^{\mathrm{ème}}$ siècle. Cette espèce, qui appartient à la famille des Astacidés, est sensible à la peste, son expansion est donc restée très limitée.

Plus récemment, c'est l'écrevisse du Pacifique (Pacifastacus leniusculus), elle aussi originaire d'Amérique du Nord, plus précisément de la côte Ouest des Etats-Unis, qui a gagné l'Europe. D'abord introduite en Suède, vers 1960, l'arrivée de l'espèce en France semble dater de 1976 (VIGNEUX et al., 1993). Elle a ensuite gagné de nombreux milieux naturels en lien avec des essais d'élevage, mais aussi grâce à des introductions occultes faites en eaux libres (LAURENT, 1983).

Considérée comme la dernière arrivée, l'écrevisse rouge de Louisiane, Procambarus Clarkii, a d'abord été introduite en Afrique, au Kenya vers 1960, puis en Espagne (LAURENT, 1989). A la faveur d'importations massives d'écrevisses vivantes à partir de ces pays (LAURENT, 1987), l'espèce est introduite en France en 1976 (LAURENT et al., 1991) où elle est librement vendue aux consommateurs. Elle ne tarde pas à faire son apparition en milieu naturel. Selon ARRIGNON et al. (1990), un certain nombre d'inconvénients comme son potentiel élevé de reproduction, qui en fait un compétiteur redoutable vis-àvis d'autres espèces d'écrevisses, mais aussi son comportement fouisseur, expliquent le statut de cette espèce en France (cf. arrêté du 21 juillet 1983 relatif à la protection des écrevisses indigènes et interdisant l'importation à l'état vivant de P.clarkii).

Enfin une nouvelle espèce nord-américaine et appartenant au genre Orconectes $(O$. juvenilis) est introduite dans le département du Doubs, signalée pour la première fois en décembre 2005 en France (DAUDEY, 2006) mais aussi en Europe.

\section{Statut réglementaire :}

Au niveau réglementaire, et contrairement à l'écrevisse à pattes grêles, les espèces originaires du continent américain, qui se comportent en porteuses saines de l'aphanomycose, sont considérées comme " susceptibles de provoquer des déséquilibres biologiques » (Art. R 232-5 du Code de l'Environnement).

Depuis la Loi «Pêche de 1984 » le transport à l'état vivant de ces espèces était interdit et la commercialisation était réglementée (Art. L432-11, L436-9, 14 et 15 du Code 
de l'Environnement). S'agissant du transport à l'état vivant, cette mesure a été abrogée par la Loi sur l'Eau du 30 décembre 2006, d'autre part les conditions de commercialisation ont été considérablement modifiées (Art. L436-9, 14 et 15 du Code de l'Environnement).

\section{L'écrevisse américaine - Orconectes limosus (Rafinesque, 1817) - Code OCL}

Parmi les écrevisses exotiques introduites, $O$. limosus est l'espèce la mieux représentée, l'espèce est signalée sur tous les bassins hydrographiques et près d'un siècle après son introduction, elle a colonisé la quasi-totalité du territoire (Corse comprise), à l'exception toutefois de la Lozère, seul département où l'espèce n'est toujours pas signalée. (Figure 4)

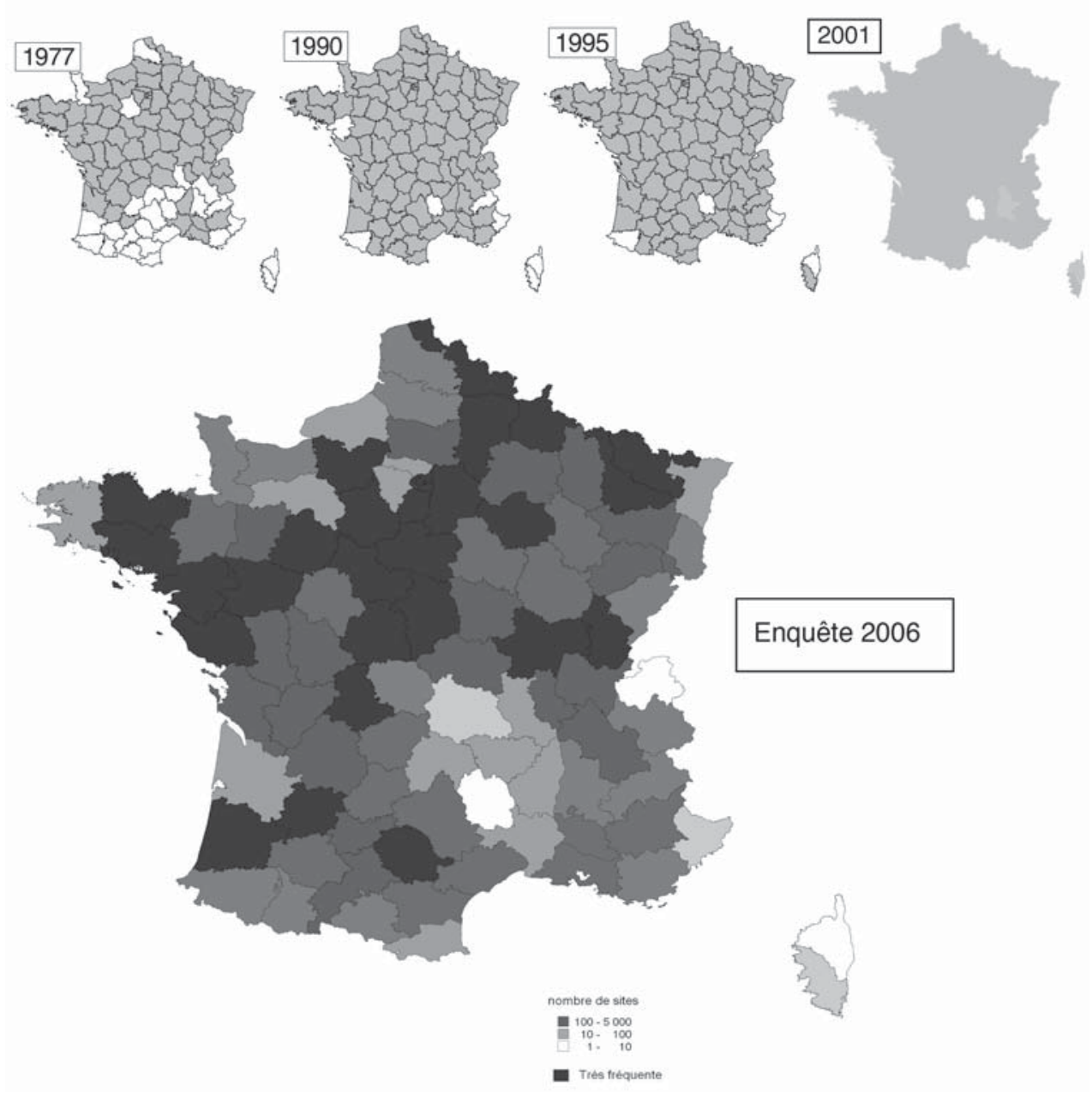

Figure 4

Evolution de la répartition de l'écrevisse américaine (Orconectes limosus). Pour les cartes des années 1977 à 2001, le signalement de l'espèce est figuré par un grisé. Pour la carte 2006, l'intensité des bleus reflète l'abondance de l'espèce à l'intérieur de chaque département, conformément à la légende.

Figure 4

Evolution of the geographical repartition of Orconectes limosus. On the 19772001 maps, the departments where the species has been observed is shaded in grey. On the 2006 map, the intensity of the blue is related to the number of observations made inside each department. 
O. limosus occupe généralement de longs linéaires de cours d'eau où les populations sont assez mal connues. Le seul indicateur quant à la situation de l'espèce sur les grands milieux est fourni par l'activité de pêche professionnelle, lorsqu'un suivi des captures est réalisé.

Cette espèce limnophile apprécie particulièrement les eaux de la seconde catégorie piscicole puisqu'elle est observée dans 95 départements, tous les grands milieux sont concernés par cette espèce dont les populations semblent toutefois se caractériser par des abondances variables, susceptibles de subir des fluctuations interannuelles importantes.

Elle est non seulement présente sur le réseau hydrographique mais aussi dans les nombreuses ballastières installées en lit majeur. Par rapport à 2001, la tendance est donc à la stabilité ; les brigades signalent cependant que les effectifs de l'espèce semblent en régression. Cette information qui reflète un sentiment général, doit cependant être prise avec précaution car OCL ne fait pas l'objet de recherches particulières.

Elle est également mentionnée dans les eaux de la première catégorie piscicole (41 départements où sa présence est certifiée et 18 où son statut reste à préciser), les populations observées présentent cependant des densités plus faibles qu'en seconde catégorie.

De nombreux plans d'eau sont également colonisés, mais le niveau d'information sur sa répartition dans ces milieux est très variable d'un département à l'autre. On observe néanmoins qu'un grand nombre de plans d'eau du domaine public, mais aussi les grands réservoirs (retenues d'alimentation des canaux). A titre d'exemple, tous les grands réservoirs de Champagne installés sur le bassin Seine sont occupés par $O$. limosus, soit plus de 10000 hectares au total.

Parmi les concurrents de cette espèce, le silure (Silurus glanis) introduit dans un grand nombre de plans d'eau du domaine public est considéré comme un des principaux responsables des diminutions de densité observées sur ces milieux.

Sur le Lac du Salagou dans l'Hérault, et suite au déversement par des pêcheurs de Procambarus clarkii, en provenance du barrage de la Rouvière (Gard), les OCL ne représenteraient plus qu'un tiers des captures d'écrevisses quatre ans après l'introduction des PCC (GALIAY, com. pers.).

D'un point de vue halieutique, la pêche de cette espèce semble assez limitée par les pêcheurs amateurs et ce, même si son prélèvement est autorisé toute l'année dans les eaux de la seconde catégorie. Elle semble davantage considérée comme une gène à l'exercice de la pêche.

Par contre, sur les grands milieux les pêcheurs professionnels exercent un prélèvement non négligeable. Sur le Rhin, par exemple, où nous disposons du suivi des captures depuis une dizaine d'années le seul pêcheur professionnel en activité annonce des captures annuelles qui peuvent être supérieures à une tonne (canal d'Alsace et Vieux-Rhin). Toutefois, ces captures se caractérisent par de grandes fluctuations interannuelles qui se traduisent par une chute importante des captures (80 à $90 \%$ ) observées notamment en 1999 et en 2005.

Plusieurs hypothèses sont avancées pour expliquer ce phénomène, mais la principale semble liée aux événements hydrologiques, les périodes de fortes crues sont généralement suivies d'une baisse importante de la population (REININGER, com.pers.). Sur la Saône en revanche, après une période d'exploitation importante dans les années 1990-1998, l'espèce a connu une régression très importante dans les captures des pêcheurs professionnels qui actuellement ne l'exploitent plus spécifiquement (BARAN, com.pers.). 
Dans le département de l'Indre (Parc de la Brenne), ce sont les activités humaines qui sont jugées responsables du recul de l'espèce, en effet, les techniques utilisées en pisciculture (chaulage) semblent constituer un frein au développement de l'espèce.

En Corse, l'espèce est signalée dans les deux départements, sur le bas Gravona (2A) et les ballastières alentours, ainsi que sur le plan d'eau d'Alzitone (2B). ASL

L'écrevisse à pattes grêles - Astacus leptodactylus (Eschscholtz, 1823) - Code

L'écrevisse à pattes grêles appartient à la famille des Astacidae, cette espèce européenne, au même titre que les espèces autochtones, est sensible à la peste de l'écrevisse. D'autre part, elle ne présente pas les inconvénients comportementaux des autres espèces importées (agressivité, creusement des berges, prolifération...), c'est pour ces différentes raisons qu'elle a intégré la liste des espèces présentes en France, elle peut donc être introduite dans toutes les eaux douces.

Les auteurs du début du siècle la décrivent dans de nombreux ouvrages, l'espèce est présentée comme résistante à la peste et l'on peut supposer que suite aux mortalités liées à l'aphanomycose, elle ait fait l'objet d'introductions. LEGER (1924), mentionne d'ailleurs l'introduction sur les marchés français d'A. leptodactylus, dite écrevisse de Galicie.

Pourtant, et selon les enquêtes nationales réalisées par le Conseil Supérieur de la Pêche, elle apparaît en France pour la première fois dans l'enquête réalisée en 1990 (26 départements) et il semble que cette espèce n'ait jamais été signalée auparavant dans les différents inventaires de la faune astacologique (ANDRE, 1960 ; LAURENT et SUSCILLON, 1962).

Entre 1990 et 2001 l'espèce va accroître son aire de répartition, elle sera recensée dans 39 (1995) puis 51 départements en 2001 (CHANGEUX, 2003). (Figure 5)

En 2006, A. leptodactylus est observée dans 41 départements, son statut est indéterminé dans 11 départements (Aisne, Alpes Maritimes, Ardennes, Aude, Cher, Creuse, Dordogne, Eure-et-Loir, Haute-Garonne, Landes, Savoie), où selon les informations transmises par les brigades l'espèce pourrait être présente dans certains plans d'eau privés. (Figure 5)

Par rapport à 2001, l'espèce n'est plus mentionnée dans 10 départements (Aube, Bouches du Rhône, Cantal, Eure, Gard, Jura, Loir-et-Cher, Lot-et-Garonne, Var, Yonne), par contre les brigades du CSP la signalent pour la première fois dans le département du Nord, le Puy-de-Dôme, la Saône-et-Loire, les Deux-Sèvres et le Val de Marne. Enfin, elle est à nouveau signalée en Ille-et-Vilaine.

L'enquête de 2006 permet de dénombrer un total de 4208 sites où l'espèce serait observée, dont 4151 correspondraient à des plans d'eau répartis sur 29 départements. On notera la forte représentation de cette espèce dans le département de la Mayenne où l'on dénombre plus de 8000 plans d'eau, dont environ la moitié serait occupée par A. leptodactylus. A lui seul ce département regrouperait $95 \%$ des sites recensés en France.

L'espèce est beaucoup plus rarement recensée sur les cours d'eau, elle serait présente sur environ 40 sites en première catégorie (14 départements) et sur une vingtaine de sites en seconde catégorie piscicole répartis dans 13 départements. Sa présence est souvent à mettre en relation avec des plans d'eau où se développe la population source.

L'espèce est utilisée à des fins d'élevage réalisé en plans d'eau privés mais ses populations sont, comme les espèces autochtones, soumises aux pressions exercées par les écrevisses exotiques. La brigade de la Creuse signale en effet que l'espèce est remplacée par l'écrevisse du Pacifique dans de nombreux plans d'eau. 

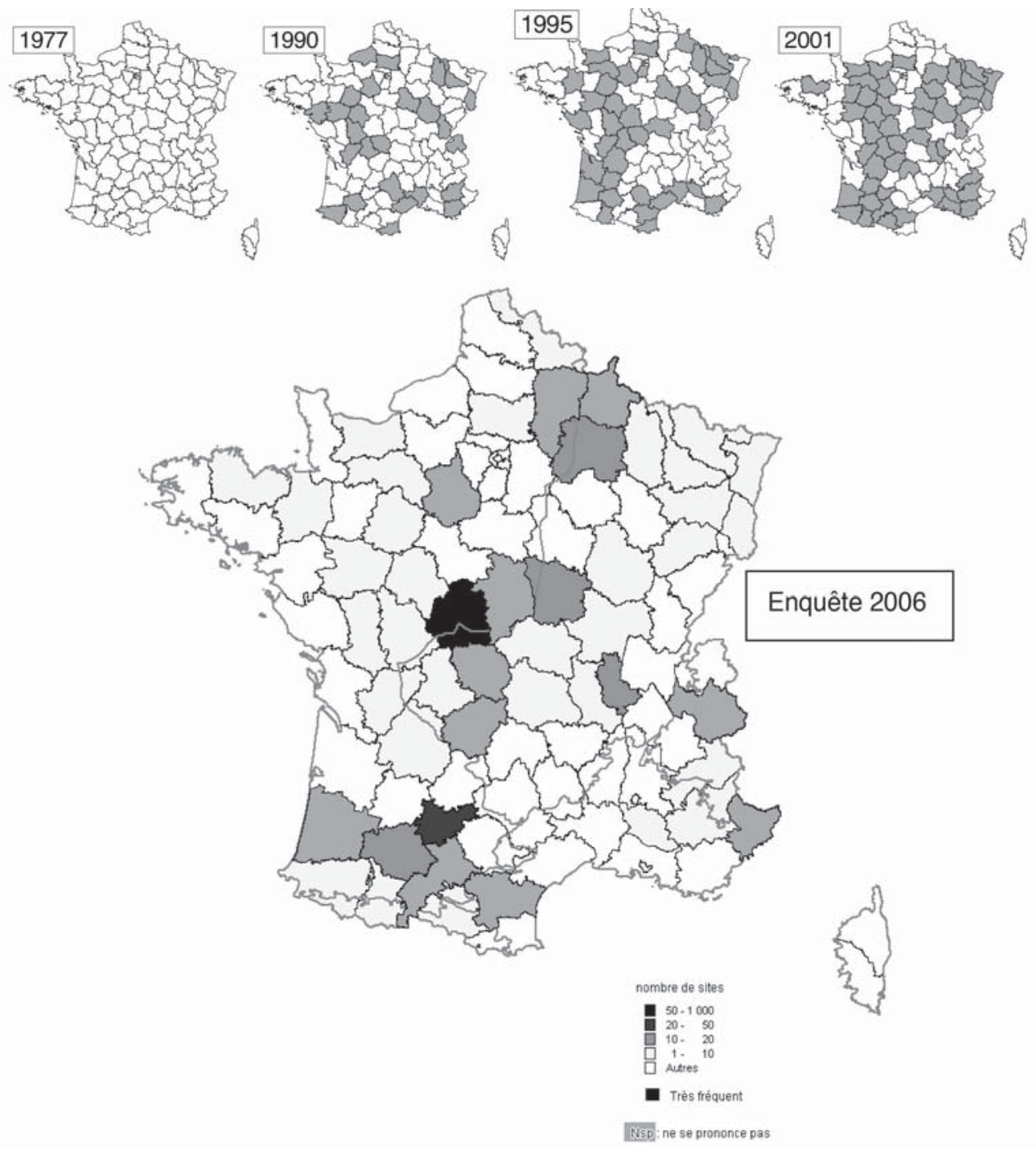

Figure 5

Evolution de la répartition de l'écrevisse à pattes grêles (Astacus leptodactylus). Pour les cartes des années 1977 à 2001, le signalement de l'espèce est figuré par un grisé. Pour la carte 2006, I'intensité des bleus reflète l'abondance de l'espèce à l'intérieur de chaque département, conformément à la légende.

Figure 5

Evolution of the geographical repartition of Astacus leptodactylus. On the 19772001 maps, the departments where the species has been observed is shadedd in grey. On the 2006 map, the intensity of the blue is related to the number of observations made inside each department.

Sa faible représentation au niveau départemental ne lui permet pas d'établir des populations pérennes et cette situation explique vraisemblablement les incertitudes qui existent autour de cette espèce discrète dont le statut est susceptible d'évoluer très rapidement au niveau local en raison notamment de sa sensibilité à l'aphanomycose.

En matière de pêche, les prescriptions adoptées pour les espèces natives s'appliquent souvent à cette espèce qui au final apparaît comme peu recherchée en raison notamment de sa faible représentation en cours d'eau. 


\section{L'écrevisse du Pacifique - Pacifastacus leniusculus (Dana, 1852) - Code PFL}

L'enquête de 1990 avait permis d'identifier plusieurs «foyers d'introduction " correspondant globalement au Limousin, à la région Rhône-Alpes et au Languedoc. Ce morcellement des populations allait permettre une évolution rapide de l'espèce sur le territoire national et depuis 2001 , on constate que la jonction est faite entre les différents sites historiques d'introduction de l'espèce. (Figure 6)

En 2006, Pacifastacus continue de progresser au niveau national, l'espèce est désormais signalée dans 73 départements, contre 61 en 2001 (+12). Les départements suivants mentionnent l'espèce pour la première fois : Aisne, Hautes-Alpes, Cher, Doubs, Drôme, Indre-et-Loire, Moselle, Pyrénées Atlantiques, Haut-Rhin, Saône-et-Loire, SeineMaritime, Yvelines, Somme, Val-de-Marne). (Figure 6)

Son statut est indéterminé dans un département (Côtes d'Armor). Elle n'est plus signalée dans deux départements, les Bouches-du-Rhône et le Calvados (CHANGEUX, 2003).

En terme de répartition, on constate que l'espèce est absente sur toute la façade atlantique (elle est signalée en Loire Atlantique en étangs privés, LAURENT, com.pers.) et en Corse.

La carte de répartition permet également de constater que les populations sont très abondantes dans le centre de la France. L'espèce est rencontrée dans différents types de milieux et si les cours d'eau à vocation salmonicole semblent constituer son milieu de prédilection (52 départements), elle est également signalée dans les eaux de la seconde catégorie (34 départements) et dans de très nombreux plans d'eau (34 départements).

Environ 1000 sites sont recensés au niveau national en 2006, soit $57 \%$ sur les cours d'eau à vocation salmonicole et $15 \%$ sur le domaine cyprinicole. Les plans d'eau représentent $28 \%$ des sites, mais ce chiffre est sous-évalué en raison de l'absence d'informations sur ces milieux dans un grand nombre de départements.

Les brigades départementales indiquent que l'espèce est en pleine expansion dans de nombreux départements, cette tendance se manifeste notamment par l'apparition de populations mais aussi par son extension vers l'aval ou vers l'amont des bassins versants en cours de colonisation ( $2 \mathrm{~km}$ par an de progression par dévalaison sur un cours d'eau du Morvan dans la Nièvre, et $3 \mathrm{~km}$ par an par montaison dans la Haute-Marne).

Ainsi, c'est précisément dans les départements de la Creuse, où des mortalités massives d'écrevisses à pieds blancs ont été observées, et de la Haute-Vienne, où cette même espèce est désormais considérée « à l'état de population relictuelle » que l'espèce semble la plus abondante, les brigades la mentionnent partout sur les eaux de la première et de la seconde catégorie.

En terme de compétition, $P$. leniusculus concurrence surtout l'écrevisse à pieds blancs, elle constitue une menace sérieuse pour les sites et les populations de cette espèce, à la fois parce qu'elle est plus compétitive sur le plan biologique, mais aussi parce qu'elle est résistante à la peste des écrevisses. Une étude réalisée en Haute-Marne (DRUART et al., 2007), sur un bassin, versant où PFL a été observée pour la première fois en 1996, a permis de mettre en évidence ses capacités à coloniser rapidement le milieu, à concurrencer et à éliminer une espèce native, en l'occurrence l'écrevisse à pieds blancs. Autre exemple, dans la Nièvre, le suivi de plusieurs petits affluents dans le Morvan a montré « un remplacement » de l' écrevisse à pieds blancs par l'écrevisse signal dans les parties aval de ces ruisseaux, les populations indigènes ne se maintenant que dans la zone apicale. Quelques particularités de non colonisation dans des cours d'eau à régime thermique très froid sont également signalées, en Bourgogne notamment (BARAN, com.pers.). 
Les informations transmises montrent qu'elle entre également en concurrence avec d'autres espèces, ainsi, PFL concurrence fortement l'écrevisse à pattes rouges pour l'éliminer progressivement en dehors de toute manifestation d'ordre pathologique (LAURENT, NICOLAS et PARIS, 1997). II en est de même lorsqu'elle est en concurrence avec l'écrevisse à pieds blancs (HOLDICH et DOMANIEWSKY, 1995). La plupart des auteurs aujourd'hui considèrent que PFL est dangereuse pour les écrevisses autochtones européennes car sa compétitivité écologique et éthologique est nettement supérieure à court et à long terme. (CUKERZIS, 1977 ; HOLDICH et al, 1995 ; NEVEU, 2006). Elle est considérée comme une espèce invasive.

Le département de la Creuse observe que l'écrevisse du Pacifique remplace l'écrevisse à pattes grêles dans de nombreux plans d'eau.

Cette espèce fait l'objet d'un engouement certain et les activités à l'origine de sa dispersion sont diverses (restauration, pisciculteurs, plans d'eau de loisirs, pêche), elle fait d'ailleurs partout où elle est présente, l'objet d'une recherche assidue à la fois par les pêcheurs amateurs mais aussi par les pêcheurs professionnels (cas du Léman). Dans l'Hérault, la brigade signale que la dissémination de l'espèce peut, en partie, être liée à certains pêcheurs, très intéressés par la pêche de cette espèce.

\section{Le cas particulier du Lac Léman}

L'écrevisse du Pacifique est arrivée dans le Lac Léman en 1979. Aujourd'hui, l'espèce est considérée comme "envahissante » dans le lac Léman (cf. la lettre du Léman $n^{\circ} 28$ de juin 2004, Bulletin d'information de la Commission Internationale pour la Protection des Eaux du Léman (CIPEL)), elle représente néanmoins une ressource économique non négligeable pour la pêche professionnelle car plusieurs tonnes sont capturées annuellement dans le lac (DUBOIS et al., 2003).

D'après ces mêmes auteurs, la pêche annuelle en 2001 atteignait 56 tonnes mais la récolte totale dans l'avenir devrait se situer entre 200 et 300 tonnes par an s'il n'apparaît aucune évolution imprévisible défavorable.

L'écrevisse du Pacifique possède le statut " d'espèce susceptible de provoquer des déséquilibres biologiques " selon les dispositions du Code de l'Environnement. Le Décret $n^{\circ} 2002-405$ du 20 mars 2002 relatif à la pêche dans le Lac Léman fixe les conditions de pêche et de transport pour les écrevisses américaines du Léman, selon certaines conditions fixées par un arrêté préfectoral, ces écrevisses, qui présentent un risque réel pour l'environnement et plus particulièrement pour les dernières populations d'écrevisses indigènes, sont expédiées vivantes dans toute la France sous la dénomination « écrevisses du Léman ».

Au cours de l'enquête, plusieurs brigades signalent la présence de ces écrevisses en provenance du Léman. Elles sont par exemple mentionnées dans le Doubs, le Jura, les Vosges (COLLAS et al., 2005b), avec de fortes présomptions de transfert par des particuliers à partir des lieux de vente, et d'introduction dans le milieu naturel.

\section{L'écrevisse rouge de Louisiane - Procambarus clarkii (Girard, 1852) - Code PCC}

L'enquête réalisée en 2006 confirme la progression constante de cette espèce qui est désormais recensée dans 61 départements. En une quinzaine d'années, l'espèce a colonisé plus de la moitié du territoire national, confirmant par la même son extraordinaire capacité d'expansion. (Figure 7)

Par rapport à 2001, PCC gagne donc 14 nouveaux départements (Aisne, Allier, Ariège, Aube, Calvados, Côte d'Or, Jura, Maine-et-Loire, Manche, Pyrénées-Orientales, Rhône, Saône-et-Loire, Yvelines, Somme). On constate également que l'espèce n'est plus mentionnée dans les départements suivants : Isère, Essonne, Hauts-de-Seine, SeineSaint-Denis et Bas-Rhin), dans ces départements, elle avait probablement été signalée par erreur en 2001. 

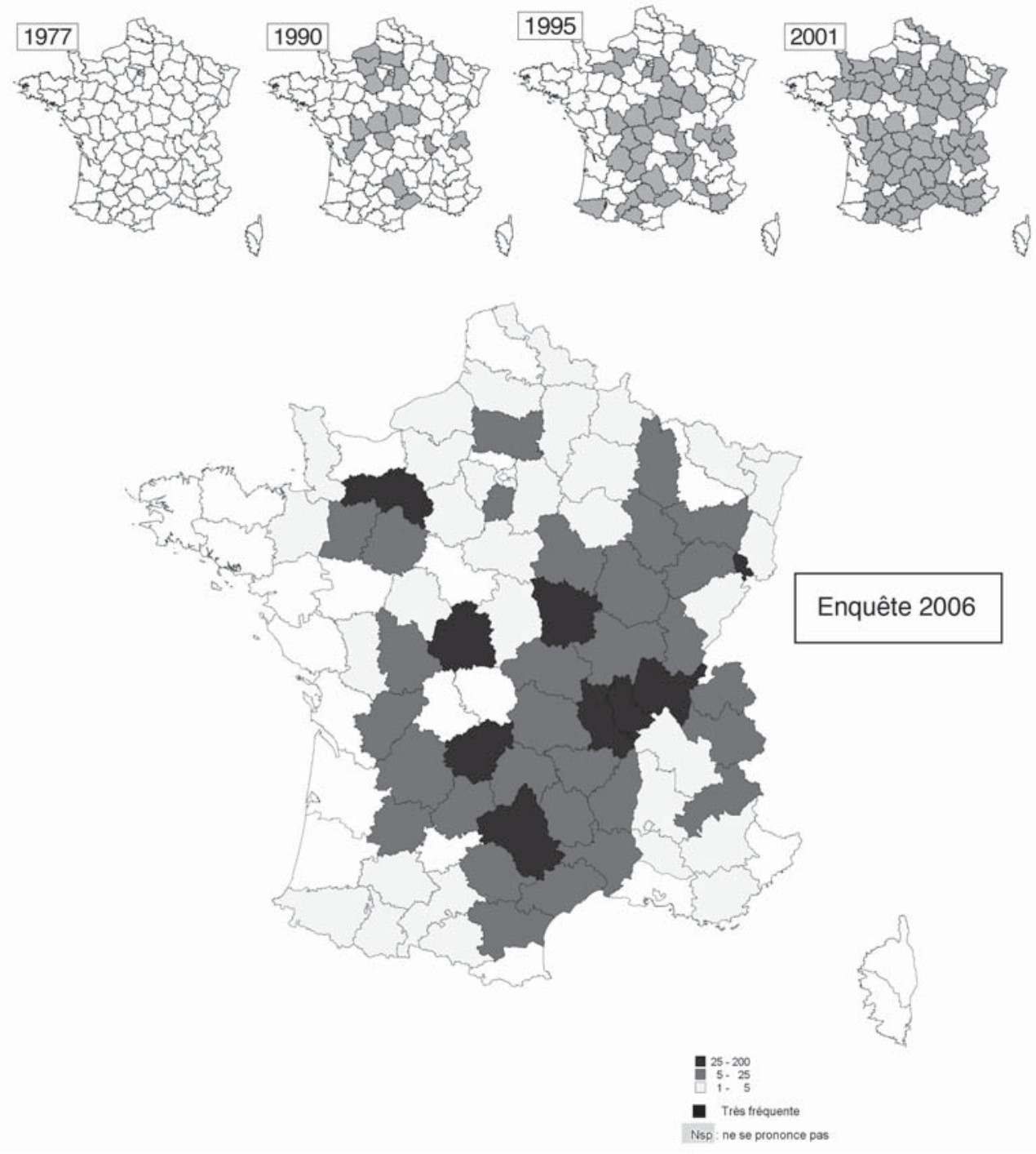

\section{Figure 6}

Evolution de la répartition de l'écrevisse Signal (Pacifastacus leniusculus). Pour les cartes des années 1977 à 2001, le signalement de l'espèce est figuré par un grisé. Pour la carte 2006, l'intensité des bleus reflète l'abondance de l'espèce à l'intérieur de chaque département, conformément à la légende.

\section{Figure 6}

Evolution of the geographical distribution of Pacifastacus leniusculus. On the 1977 to 2001 maps, the departments where th especies has been observed is shaded in grey. On the 2006 map, the intensity of the blues shows the abundance of the species inside each department.

Le statut de Procambarus clarkii reste à préciser dans les départements des Côtes d'Armor, de la Haute-Saône et du Var, dans ce dernier l'espèce était signalée en 2001, sa présence non vérifiée durant la période 2001-2006, est toutefois supposée.

La carte de répartition de l'espèce indique de fortes densités sur toute la façade atlantique, de la Loire-Atlantique, jusqu'au département des Landes, mais aussi sur la 
façade méditerranéenne (Hérault, Gard, Bouches-du-Rhône). Dans ces départements pionniers, les brigades signalent un tassement des effectifs sur les premiers sites colonisés, mais constatent parallèlement l'explosion des populations sur les nouveaux sites (Gironde, Charente-Maritime, Loire-Atlantique, Deux-Sèvres). En Gironde une observation sur le questionnaire indique "qu'il vaudrait mieux chercher les milieux où l'espèce n'est pas encore présente », traduisant ainsi la forte représentation de PCC.

Globalement l'enquête de 2006 fait apparaître plusieurs tendances :

- une augmentation de l'aire colonisée, se traduisant notamment par une progression continue de l'espèce notamment vers le nord et le centre du pays, l'extrémité est restant actuellement vierge de fréquentation,

- une progression de l'espèce vers les zones amont des hydrosystèmes. Cette espèce est sans doute celle qui présente le spectre écologique le plus large, alors qu'on la décrivait surtout inféodée aux " eaux chaudes » de la zone potamique, elle est capable de coloniser et d'occuper des milieux variés allant de la zone à truite aux étangs côtiers en passant par les marais. Elle est même mentionnée dans certains milieux temporaires (Loire Atlantique) et dans des milieux soumis aux marées (Pyrénées atlantiques).

Ainsi, PCC est observée dans les eaux de la première catégorie (74 sites signalés dans 17 départements) et sa tendance à se développer sur les zones apicales est mentionnée dans plusieurs départements (Bouches-du-Rhône, Manche). On constate également que lors de l'enquête précédente deux départements seulement signalaient l'espèce dans les eaux de première catégorie piscicole.

Elle est abondante dans les eaux de la seconde catégorie (511 sites en seconde catégorie pour 37 départements). Au total 580 sites sont signalés sur les eaux courantes dans 45 départements.

L'espèce est également abondante dans les plans d'eau, où le niveau d'information apparaît très variable, elle est signalée dans 38 départements pour un total de 427 plans d'eau. Ce chiffre, bien entendu, sous-estime largement la situation de l'espèce dans ces milieux ; 15 départements ne se prononcent pas sur le statut de PCC en plans d'eau.

Environ 1000 sites sont recensés au niveau national en 2006, soit $58 \%$ sur les cours d'eau dont $7 \%$ sur les ruisseaux à vocation salmonicole et $51 \%$ sur le domaine cyprinicole. Les plans d'eau représentent $42 \%$ des sites, mais ce chiffre est sousévalué en raison de l'absence d'informations sur ces milieux dans un grand nombre de départements.

L'activité halieutique autour de cette espèce peut être qualifiée d'active, elle génère dans de nombreux départements où l'espèce est abondante, un véritable engouement. Cette ressource fait en effet l'objet d'un prélèvement à la fois par les pêcheurs amateurs, mais aussi par les pêcheurs professionnels qui la commercialisent en « mort-frais " sur le Lac de Grand Lieu (BRAMARD C., Com.pers.).

Cette activité peut être considérée comme un vecteur de dispersion de l'espèce (Charente-Maritime). BRAMARD M. (com.pers.) signale également que les vacanciers du bord de mer n'hésitent pas à rapporter chez eux cette espèce et à l'introduire dans des mares, bassins... d'où elles s'échappent rapidement pour gagner les eaux libres.

\section{Orconectes Juvenilis (Hagen, 1870) - Code OCJ (Figure 8)}

A la faveur de prospections nocturnes réalisées conjointement par la brigade départementale du CSP et la Fédération de pêche du Doubs, une nouvelle espèce a été découverte sur le bassin du Dessoubre en 2005 (GINDRE, MOUGET, PERRINE, com.pers.). 

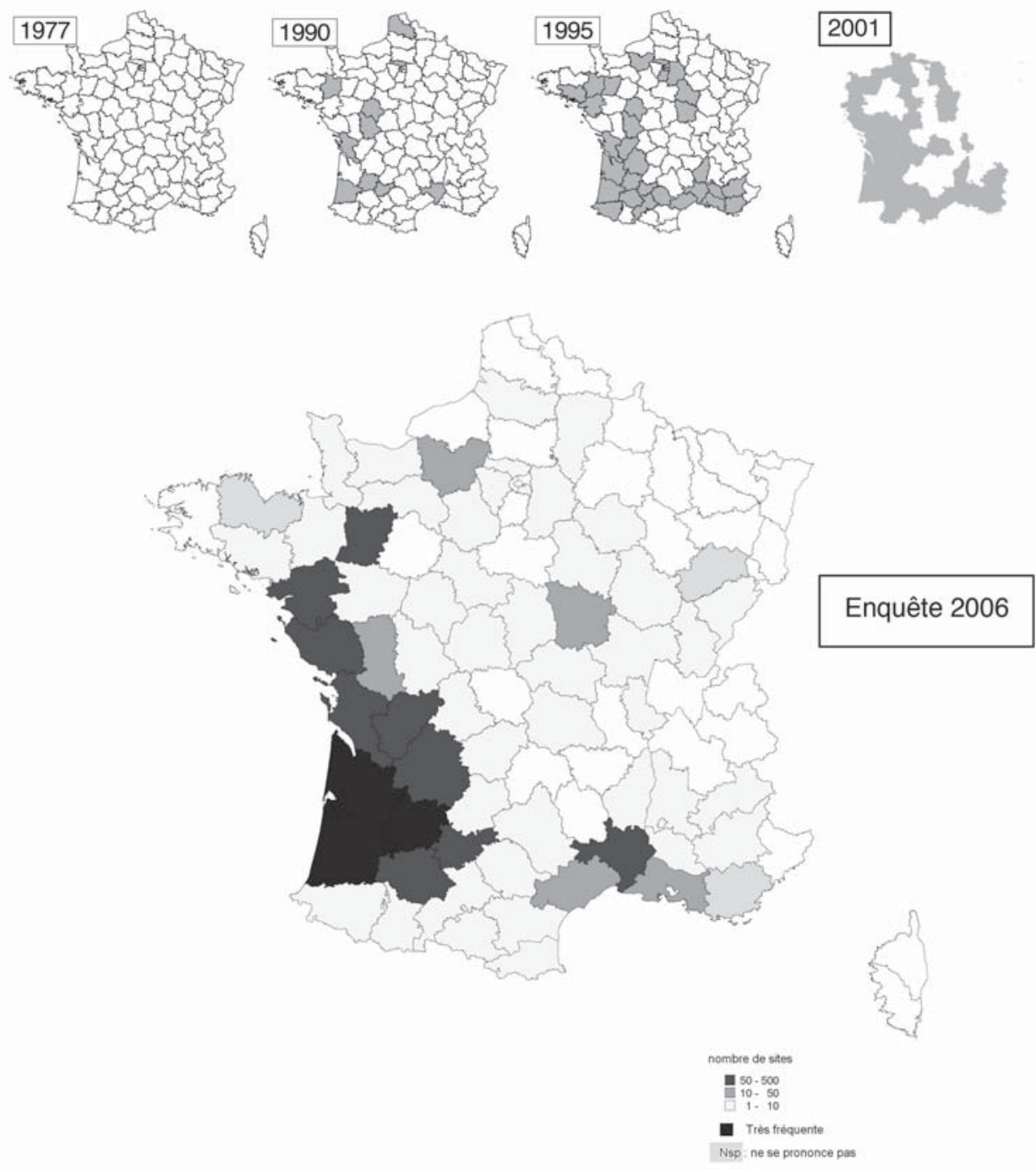

Figure 7

Evolution de la répartition de l'écrevisse rouge de Louisiane (Procambarus clarkii). Pour les cartes des années 1977 à 2001, le signalement de l'espèce est figuré par un grisé. Pour la carte 2006, l'intensité des bleus reflète l'abondance de l'espèce à l'intérieur de chaque département, conformément à la légende.

Figure 7

Evolution of the geographical distribution of Procambarus clarkii. On the 1977 to 2001 maps, the departments where the species has been observed is shaded in grey. On the 2006 map, the intensity of the blues shows the abundance of the species inside each department.

La présence et l'apparition de cette nouvelle espèce semblent liées à un restaurateur installé sur la commune de Rosureux. En effet l'écrevisse aurait été stockée dans deux bassins situés à proximité de l'établissement avant de rejoindre le Dessoubre proche (DAUDEY, 2006). L'origine et les circonstances par lesquelles cette écrevisse est arrivée en France restent toutefois méconnues à ce jour. 

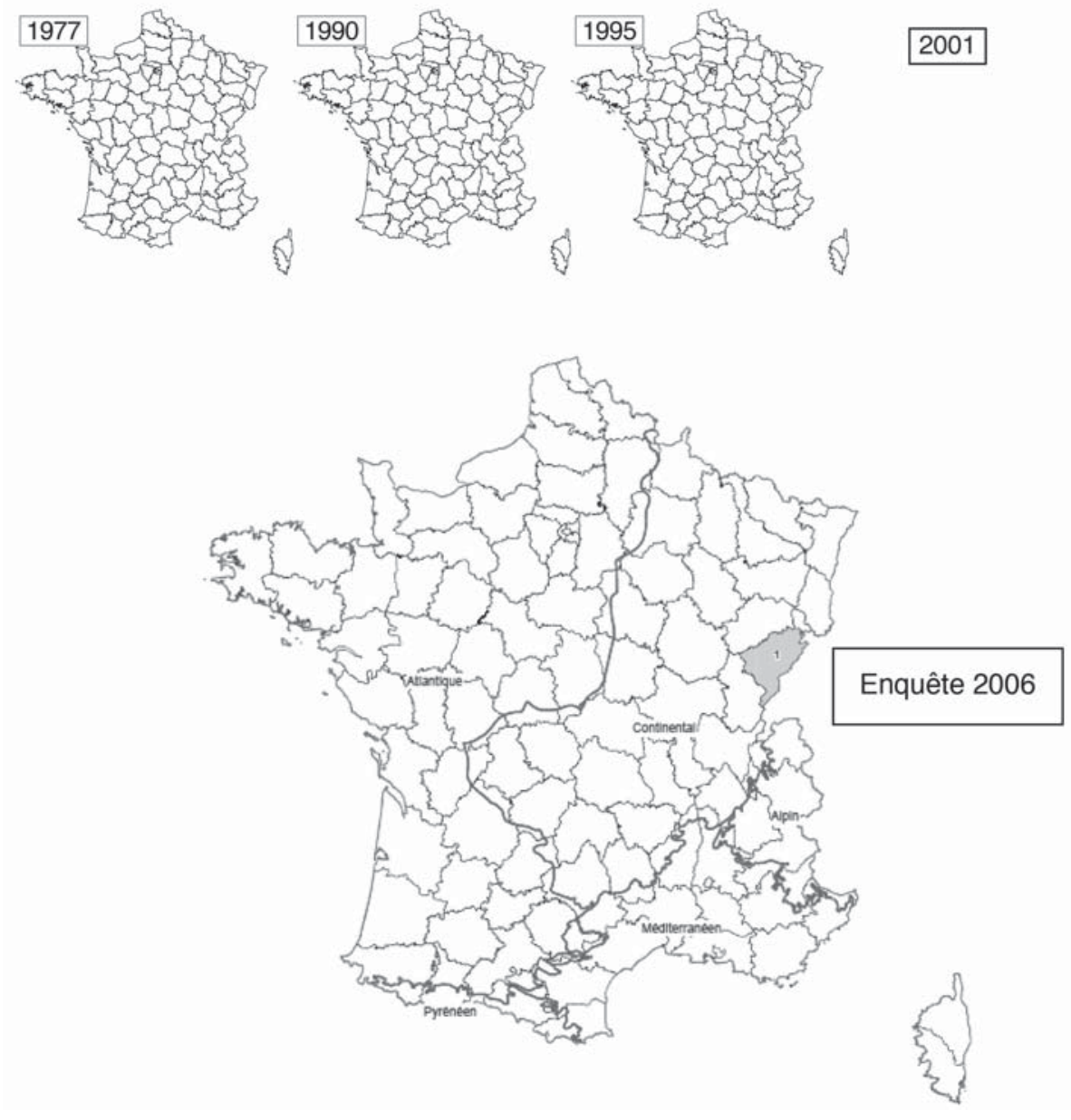

Figure 8

Evolution de la répartition d'Orconectes juvenilis. Pour les cartes des années 1977 à 2001, le signalement de l'espèce est figuré par un grisé. Pour la carte 2006, l'intensité des bleus reflète l'abondance de l'espèce à l'intérieur de chaque département, conformément à la légende.

Figure 8

Evolution of the mortalities of Orconectes juvenilis. On the 1977-2001 maps, observations of the species are marked as a grey shading. On the 2006 map, the intensity of he blue shows the abundance of the species inside the department.

Suite au signalement de cette nouvelle espèce, l'écrevisse est dans un premier temps identifiée comme Orconectes rusticus dans Atlas of Crayfish in Europe (SOUTYGROSSET et al., 2006). Mais de nouvelles déterminations réalisées aux Etats-Unis par le Professeur Chris TAYLOR, (Illinois Natural History Survey), permettent d'identifier Orconectes juvenilis (COLLAS et TAYLOR, à paraitre).

De nombreux auteurs américains ont longtemps considéré ces deux espèces comme étant très proches, mais TAYLOR (2000), élève Orconectes juvenilis au rang d'espèce.

Orconectes juvenilis est une espèce originaire d'Amérique du Nord, plus particulièrement des Etats de l'Indiana et du Kentucky. 


\section{Le point sur les mortalités}

L'enquête nationale réalisée en 2006 avait également pour objectif de vérifier auprès des brigades départementales si des mortalités d'écrevisses avaient pu être observées au cours de la période 2001/2006. Cette partie de l'enquête n'avait jamais été abordée au cours des enquêtes réalisées auparavant.

Le questionnaire permettait entre autre d'indiquer l'origine de la mortalité (pathologie, pollution...), de préciser la date à laquelle elle avait été observée, mais aussi l'espèce victime, voire l'espèce susceptible d'être à l'origine de ces phénomènes.

\section{Résultats}

Pour la période 2001/2006, 47 départements (49\%) signalent des mortalités d'écrevisses, représentant 97 cas distincts.

Cinq espèces d'écrevisses sont mentionnées (Figure 9) :

- S'agissant des écrevisses endémiques, l'écrevisse à pieds blancs représente à elle seule $89 \%$ des cas observés, cette espèce subit des mortalités dans 41 départements, dont les causes principales sont les événements climatiques observés depuis 2003 et des épizooties diverses (aphanomycose et thélohaniose). L'écrevisse à pieds rouges subit des mortalités dans les départements de la Nièvre (sécheresse) et des Vosges (aphanomycose). Cette espèce représente $2 \%$ des cas.

- S'agissant des écrevisses exotiques, elles représentent un total de neuf cas, dont 6 pour la Pacifastacus (4 dont l'origine est inconnue et deux liés à des pollutions), 2 pour la leptodactylus, et un pour la Procambarus.

\section{Répartition des mortalités par espèce Période2001-2006}

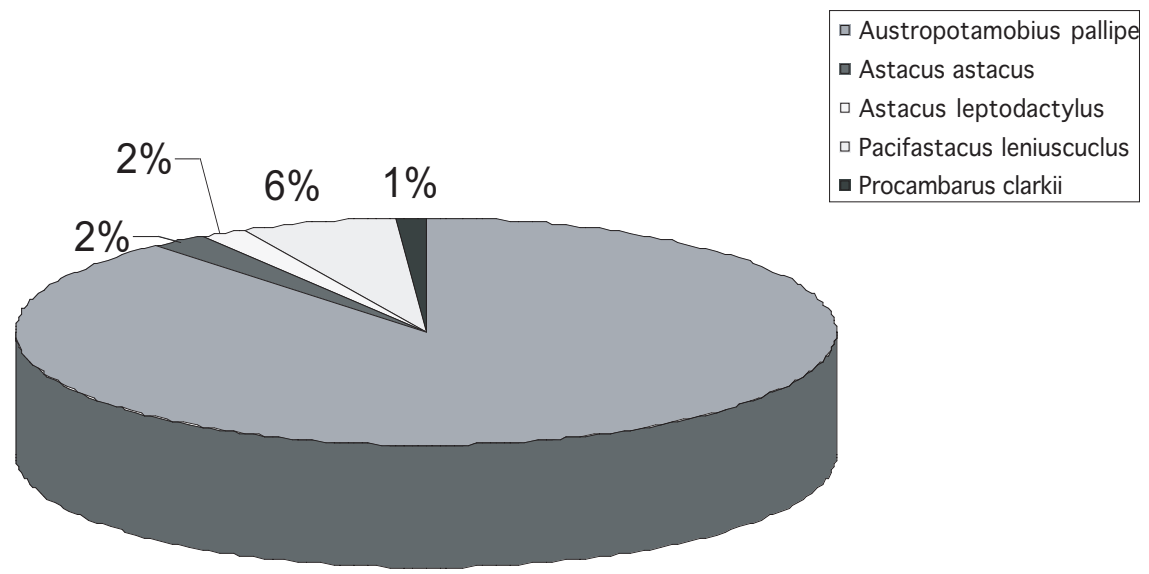

$89 \%$

\section{Figure 9}

Répartition par espèce des mortalités d'écrevisses observées en France. Période 2001-2006 - Enquête CSP

\section{Figure 9}

Repartition by species of crayfish mortalities observed in France. CSP 2001-2006 Survey. 
On peut identifier plusieurs origines aux mortalités (Figure 10), avec par ordre d'importance :

\section{Origine des mortalités d'écrevisses en France Période 2001 à 2006}

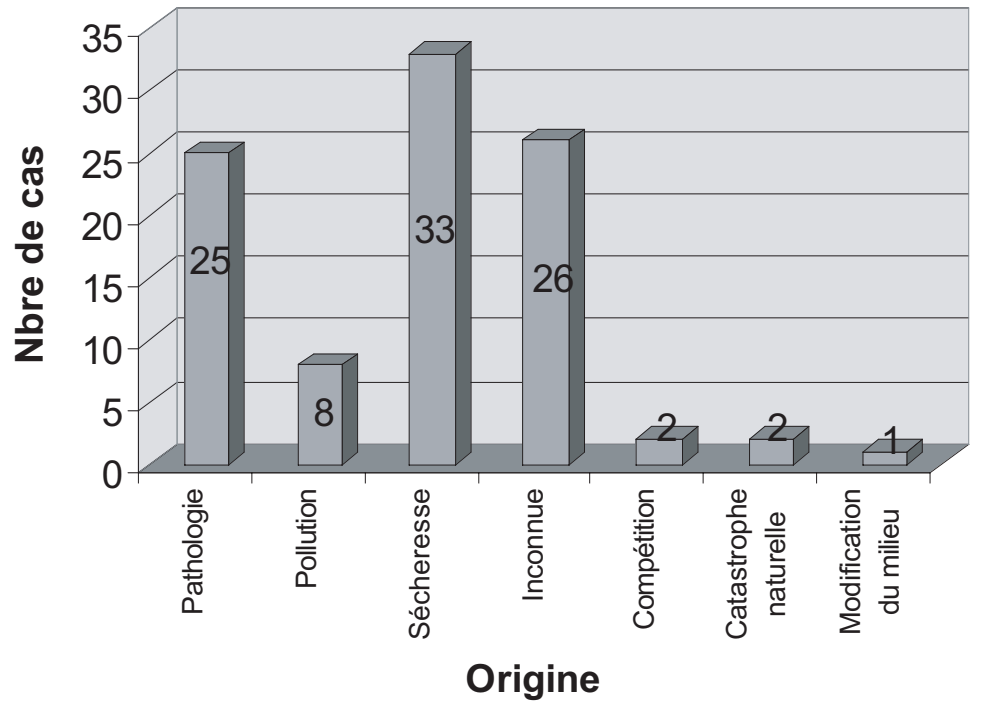

Figure 10

Origines des mortalités d'écrevisse en France Période 2001-2006 (CSP, Enquête 2006).

\section{Figure 10}

Origins of crayfish mortalities in France, 2001-2006 - (CSP, Survey 2006).

- Les événements climatiques observés entre 2003, 2005 et 2006 ont occasionné de nombreux assecs sur les ruisseaux situés en tête de bassin où les écrevisses endémiques, et plus particulièrement l'écrevisse à pieds blancs, trouvent refuge. Les mortalités liées à ces événements climatiques représentent 33 cas, soit $34 \%$ des cas de mortalité observés au niveau national. Ce phénomène a pu être observé dans 19 départements (Ain, Alpes de Hautes Provence, Aude, Aveyron, Bouches du Rhône, Calvados, Cantal, Côtes d'Armor, Gard, Gers, Hérault, Indre et Loire, Lozère, Nièvre, Hautes-Pyrénées, Pyrénées Orientales, Deux-Sèvres, Tarn et Garonne, Vienne). Les chiffres mentionnés semblent toutefois représenter un minimum, les départements du Gard et du Cantal signalent en effet plusieurs dizaines de cas de mortalités liés à la sécheresse 2003 et 2005 sans toutefois préciser les milieux affectés. D'autre part, la Corse mentionne que les populations sont affectées par la sécheresse 2003, sans préciser la nature des mortalités observées.

- 25 cas de mortalités liés à des pathologies $(25 \%)$ sont mentionnés dans 16 départements (Ardèche, Ariège, Cher, Côtes d'Armor, Creuse, Eure, Gard, Gers, Hérault, Jura, Lot, Lozère, Pyrénées Atlantiques, Hautes-Pyrénées, Deux Sèvres, Vosges). NEVEU (1998 b) mais aussi VOGT (1999) signalent un retour en force de la peste des écrevisses en liaison avec de nouvelles souches introduites par les écrevisses américaines et plus particulièrement $P$. leniusculus. Deux pathologies sont citées dans l'enquête: L'Aphanomycose ou "peste des écrevisses " et la Thélohaniose ou "maladie de la porcelaine ", chacune de ces pathologies représente dix cas, avec des mortalités plus ou moins importantes. Celles liées à l'aphanomycose sont généralement massives et rapides tandis que celles liées à la thélohaniose, sévissent sur des populations qui sont déjà affaiblies ou en situation de stress, leur importance est variable. Dans certains 

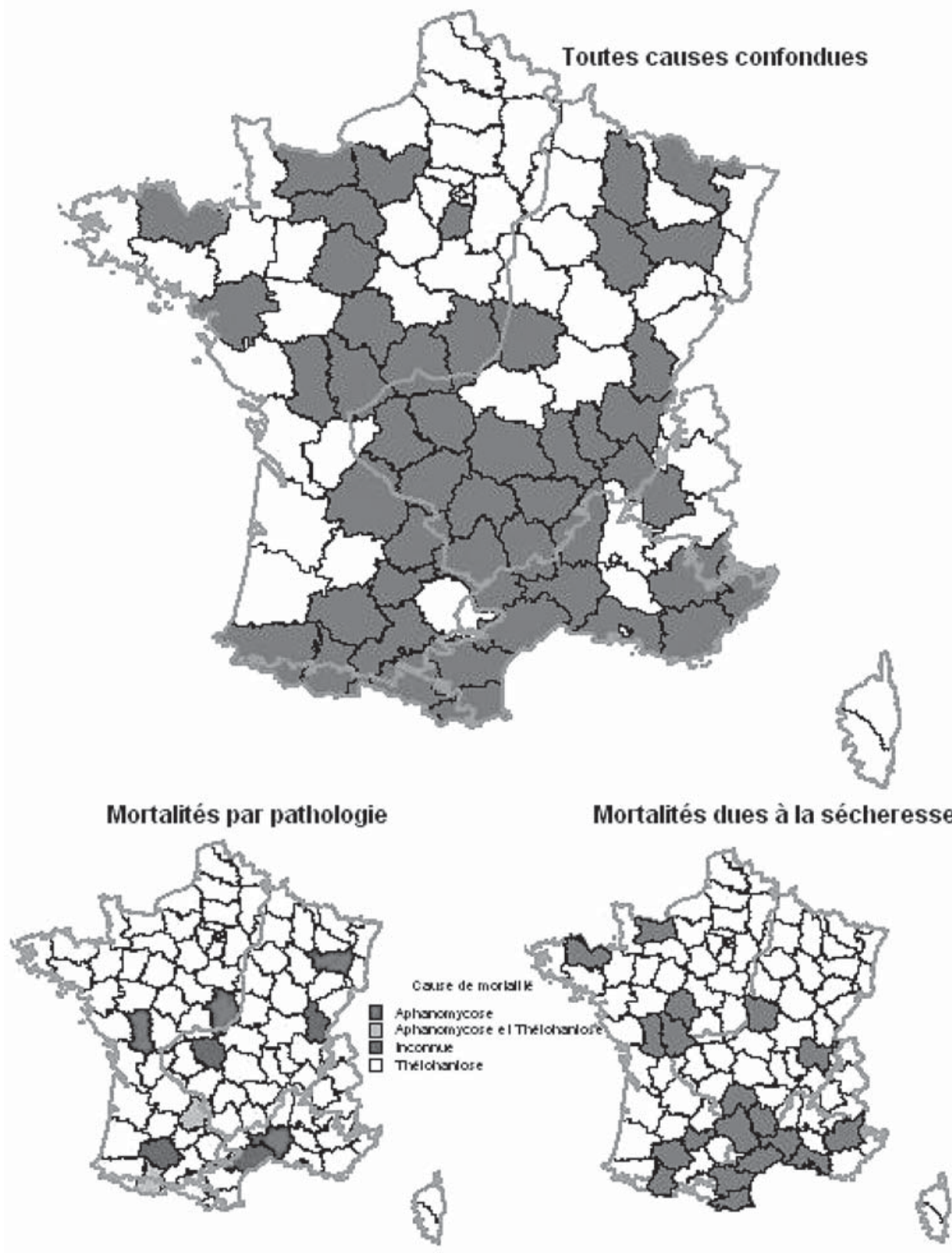

Figure 11

Carte des mortalités d'écrevisses toutes espèces confondues, durant la période 2001-2005.

Figure 11

Map of crayfish mortalities origins, all species considered, 2001-2005.

départements, ces pathologies ont fait l'objet d'un diagnostic en laboratoire, c'est le cas dans les Deux Sèvres (NEVEU et BACHELIER, 2003), dans les Vosges (COLLAS et SALEK, 2002) dans le Lot (BD47, com.pers.) ou le Calvados (ANONYME, 2002), où la présence de foyers d'aphanomycose a pu être confirmée. Signalons que dans le département de la Creuse, des mortalités massives ont été observées en 2001 sur l'ensemble des sites abritant des APP, ces mortalités ont abouti à la disparition de l'espèce dans le département. Enfin pour cinq de ces mortalités, la pathologie est restée inconnue. 
- 25 cas des mortalités signalées ont une origine inconnue. Elles sont vraisemblablement liées à des pathologies, mais les investigations réalisées sur le terrain ou les circonstances de ces mortalités n'ont pas permis d'en déterminer la cause précise.

Parmi les autres signalements, 8 cas ont pour origine des pollutions diverses (station d'épuration, traitements à l'aide de phytosanitaires...), tandis que les atteintes à l'habitat représentent seulement un cas (Gard).

Paradoxalement, alors que de nombreuses brigades signalent la forte expansion des écrevisses exotiques et notamment de Pacifastacus et de Procambarus, la compétition interspécifique ne représente que deux cas de mortalités. L'un concerne l'écrevisse du Pacifique et l'écrevisse à pieds blancs dans le département de la Haute-Marne, où un suivi de l'expansion de l'espèce a été mis en place depuis plusieurs années (DRUART et al., 2007). Le second concerne l'écrevisse à pieds blancs et l'écrevisse rouge de Louisiane dans le département du Tarn et Garonne où la colonisation des milieux par cette espèce invasive est très active depuis environ cinq ans.

On peut donc, en liaison avec les résultats de l'enquête nationale 2006, qui montrent une nouvelle fois l'expansion des écrevisses introduites au détriment des espèces endémiques, penser que la compétition interspécifique est largement sous-estimée. Ce phénomène reste toutefois difficile à apprécier, les zones de conflit et de contact entre espèces natives et exotiques sont assez rares et peu étudiées. Ainsi, un délai plus ou moins long peut exister entre le moment de la disparition de l'espèce endémique et l'apparition ou le signalement de l'espèce introduite. La rubrique " mortalités " que les brigades renseignaient pour la première fois en 2006 ne semblent donc pas constituer un outil satisfaisant pour caractériser les phénomènes de compétition inter-spécifique, le remplacement des espèces est le plus souvent discret et avec des échéances variables en terme de temps.

\section{CONCLUSION - DISCUSSION}

Les tendances observées au cours des enquêtes précédentes (VIGNEUX, 1980 ; ARRIGNON, 1990 ; CHANGEUX, 1996 et 2003) semblent se confirmer avec la réalisation de l'enquête nationale sur la répartition des écrevisses réalisée en 2006.

Les trois écrevisses indigènes continuent de régresser quand, parallèlement, plusieurs écrevisses exotiques montrent une capacité remarquable à coloniser les différents habitats disponibles et à concurrencer les dernières populations d'écrevisses natives. Cette expansion concerne principalement l'écrevisse américaine, même si elle a déjà colonisé la quasi-totalité des eaux cyprinicoles, l'écrevisse du Pacifique qui s'avère représenter un redoutable concurrent pour nos espèces indigènes, mais aussi l'écrevisse rouge de Louisiane dont la répartition évolue très rapidement et qui possède des caractéristiques biologiques lui permettant de coloniser des milieux très variés.

La situation des écrevisses indigènes doit désormais être considérée comme préoccupante, voire alarmante ; en effet, l'enquête montre que l'écrevisse des torrents, l'écrevisse à pieds blancs et l'écrevisse à pattes rouges sont cantonnées sur des secteurs exempts de perturbation et qu'elles ont disparu de leurs habitats de prédilection pour se réfugier sur des secteurs apicaux peu en rapport avec leurs exigences écologiques. Ces secteurs se caractérisent généralement par un habitat préservé (qualité d'eau et milieu physique). Les populations sont toutefois fragilisées car elles se trouvent sur des milieux sensibles, en dehors de leur preferendum typologique.

Cette tendance est observée au niveau national au travers des nombreux programmes de connaissance départementaux ou régionaux mis en œuvre à l'initiative des brigades du CSP ou des fédérations de pêche. 


\section{Le bilan réalisé en 2006 est frappant :}

- l'écrevisse des torrents n'est plus représentée que sur deux sites en France, totalement isolés en tête de bassin,

- l'écrevisse à pattes rouges subsiste dans quelques plans d'eau privés (62 \% des sites), généralement implantés sur de petits ruisseaux forestiers, mais aussi grâce aux efforts déployés par quelques astaciculteurs privés. Sa répartition sur les cours d'eau est en effet très limitée et souvent en relation avec l'existence d'un plan d'eau (COLLAS, 1995). Les milieux désertés par l'espèce pour des raisons multiples, sont aujourd'hui colonisés par les écrevisses exotiques plus tolérantes, qui prospèrent et continuent leur progression vers l'amont,

- l'écrevisse à pieds blancs est mentionnée dans 76 départements. On remarque que son statut est fragile mais aussi extrêmement variable suivant les départements ou les régions et qu'elle peut désormais être considérée comme disparue ou en voie de disparition dans un grand nombre de départements. Cette espèce emblématique est encore présente sur environ 2500 à 3000 sites, avec une zone privilégiée dans le centre et dans le Sud-Est de la France.

Le département de la Moselle est le seul département où les trois espèces endémiques ont été recensées.

La concurrence engendrée par les écrevisses exotiques ne constitue pas la seule menace pour les peuplements indigènes, elle se manifeste néanmoins au travers de Pacifastacus leniusculus, mais aussi de Procambarus clarkii, signalée dans 13 nouveaux départements et qui semble gagner à son tour la zone à truite. On peut également citer les pathologies, comme l'aphanomycose ou la thélohaniose qui sont signalées dans cette enquête dans différentes régions de France, souvent en relation avec l'apparition ou la présence des écrevisses exotiques (NEVEU, 1998a ; NEVEU et BACHELIER, 2003).

Les informations fournies par les agents du Conseil Supérieur de la Pêche identifient également une dégradation de l'habitat des dernières populations indigènes. Les événements climatiques enregistrés pendant la période 2003/2006 se sont traduits par une modification profonde des conditions hydrauliques sur de nombreuses têtes de bassin. L'assèchement de ces milieux refuges, observé dans une vingtaine de départements, a causé la disparition de nombreuses populations d'écrevisses, elle est à mettre en relation avec le changement global que représente le réchauffement climatique. Ainsi selon un rapport de l'Organisation de coopération et de développement économiques (OCDE, à paraître) les Alpes sont particulièrement sensibles aux changements climatiques et le réchauffement récent y a été près de trois fois supérieur à la moyenne mondiale. Les années 1994, 2000, 2002, et 2003 ont été les plus chaudes dans les Alpes au cours des cinq cents dernières années (d'après des reconstructions de haute résolution du climat de la région depuis 1500 après $\mathrm{J}-\mathrm{C}$.).

Entre la progression des espèces exotiques, et la perte de leurs derniers habitats, l'avenir des écrevisses natives apparaît de plus en plus incertain. L'écrevisse à pieds blancs semble la plus concernée puisqu'elle représente $89 \%$ des cas de mortalités observées en France entre 2001 et 2006 (la sensibilité particulière des agents pour cette espèce peut cependant influencer ce résultat).

Ce sentiment est renforcé par l'apparition en 2005 d'une nouvelle espèce en provenance d'Amérique du Nord (Orconectes juvenilis), qui a déjà réussi à gagner les eaux libres, mais aussi par les récentes évolutions de la législation qui autorisent désormais le transport à l'état vivant des "espèces susceptibles de provoquer des déséquilibres biologiques ". L'abrogation de l'article L432-11 du Code de l'Environnement par la Loi du 30 décembre 2006, va en effet à l'encontre des dispositions de la Loi du 2 février 1995 
relative au renforcement de la protection de l'environnement et qui affichait comme objectif de contrôler les effets des introductions qui se sont avérées dommageables pour la faune et la flore sauvages. Pour les écrevisses, LAURENT (1997) considérait que l'interdiction du transport à l'état vivant devait être impérativement maintenue.

La préservation des dernières populations d'écrevisses indigènes en France doit faire l'objet d'un plan national pour les écrevisses, à l'exemple de celui proposé par la Suisse (STUCKI et ZAUGG, 2006 ; HEFTI et STUCKI, 2006). Ce plan définit notamment des mesures de protection destinées aux espèces indigènes :

- protection de I'habitat : à titre d'exemple la révision du SDAGE Adour Garonne prévoit de prendre en compte des critères écologiques forts pour la préservation de l'écrevisse à pieds blancs. Ainsi, la présence de cette espèce constitue un argument d'opposition systématique aux travaux soumis à déclaration quand ils sont susceptibles de détruire ou de dégrader l'habitat. D'autre part, le programme LIFE «Tête de Bassin et Faune Patrimoniale associée » en cours actuellement dans les régions Bourgogne et Franche-Comté permet de conduire des actions coordonnées dans le domaine de la connaissance des espèces indigènes mais surtout dans la restauration des populations et des habitats. Des opérations de renaturation de ruisseaux, d'effacement de plans d'eau et de réintroduction ont lieu, elles devraient permettre d'enrichir le bagage technique dans le domaine de la conservation des espèces autochtones d'écrevisses.

- définition des sites regroupant les principales populations d'écrevisses autochtones,

- définition des populations sources dans le cadre d'un programme de réintroduction, conservation et exploitation raisonnée des populations.

Ce dispositif prévoit également des mesures de lutte destinées aux espèces non indigènes :

- contrôle d'expansion des populations par des campagnes d'élimination coordonnées,

- information et sensibilisation du public sur les dangers liés à la propagation des espèces exotiques dans le milieu naturel. C'est dans cet objectif que le Conseil Supérieur de la Pêche a publié une plaquette d'information sur les risques liés à l'introduction des espèces exotiques (COLLAS et al., 2006),

- établir des conditions d'exploitation et de commercialisation des écrevisses invasives, avec des cahiers d'exploitation stricts,

- suivre l'évolution des pathologies et plus particulièrement de l'aphanomycose. En effet, le diagnostic de cette maladie a subi une évolution importante et peut désormais être établi d'une manière plus sûre qu'autrefois. L'apport de nouvelles techniques moléculaires en usage en Espagne et en Suède permet aujourd'hui de déterminer quelle espèce américaine est à l'origine de l'épizootie, mais aussi de déterminer les populations saines et celles porteuses de la pathologie. Actuellement, des mortalités massives sont signalées (département de la Creuse) sans qu'aucune hypothèse sur leur cause ne puisse être avancée.

Dans la continuité des travaux entrepris et dés 2007, l'Onema va donc poursuivre son effort de connaissance sur la répartition des écrevisses. Un travail important de connaissance est actuellement en cours en région Bourgogne, et des enquêtes sont en cours dans plusieurs départements (Alpes-de-Haute-Provence, Aude, Bouches-duRhône, Drôme, Eure, Haute-Marne, Orne, Pyrénées-Atlantiques, Rhône, Haute-Saône, Savoie, Vosges, Vendée...). Dans plusieurs autres elles ont déjà abouti à la réalisation d'atlas de répartition. 
Ces travaux nécessitent néanmoins une harmonisation au niveau national qui pourra donner naissance à la création d'une base de données nationale sur les écrevisses. Ce programme, préalable indispensable, permettra entre autres, de préciser le statut de chaque espèce à l'échelle des différents bassins versants (unité de gestion) et non plus à l'échelle d'un département.

La structure actuelle de l'enquête et la notion de présence/absence par département rendent impossible l'établissement de cartes de répartition par bassin. Cette orientation permettrait en terme de gestion de définir les zones où les actions doivent être menées en priorité.

Parallèlement, l'enquête montre qu'un effort important doit être entrepris sur la connaissance des espèces non indigènes et plus particulièrement sur leur répartition dans les plans d'eau où les données sont très fragmentaires. L'enquête 2006 , comme les précédentes, montre la difficulté de connaissance sur ces milieux, qui constituent pourtant un vecteur essentiel de propagation et de dispersion des espèces exotiques vers les eaux libres.

\section{REMERCIEMENTS :}

Les auteurs remercient les correspondants "écrevisses " dans les différentes délégations régionales du CSP : Jean Maxence Ditche, Frédéric Villette (Compiègne), Arnaud Richard (Rennes), Michel Bramard (Poitiers), François Vanceysselle (Lyon), René Martin (Clermont-Ferrand), Eric Galliay (Toulouse), Fabrice Laval (Montpellier), Philippe Baran (Dijon), ainsi que l'ensemble des brigades départementales du Conseil Supérieur de la Pêche.

\section{BIBLIOGRAPHIE}

ANDRE M., 1938a. Sur l'acclimatation en France d'une écrevisse américaine du genre Cambarus (C. affinis Say). Bull. Fr. Pêche Piscic., 113:18-21.

ANDRE M., 1938b. Sur l'acclimatation en France d'une écrevisse américaine du genre Cambarus (C. affinis Say). Bull. Fr. Pêche Piscic., 114 :42-48.

ANDRE M., 1960. Les écrevisses françaises. Editions Lechevallier, 293 p.

ANDRE M., LAMY E., 1935. Les écrevisses de France, 89 pages, 7 figures. Chez les auteurs, 61 rue de Buffon, Paris $\left(5^{\circ}\right)$.

ANONYME, 2002. Nouveau cas d'Aphanamycose dans le Calvados ; L'Astaciculteur de France, $71: 21$.

ARRIGNON J., 1990. L'enquête nationale sur les écrevisses, une réglementation inadaptée. Eaux libres, 2.

ARRIGNON J.C.V., HUNER J.V., LAURENT P.J., 1990. L'écrevisse rouge des marais. Le Technicien d'Agriculture Tropicale, Editions Maisonneuve et Larose, Paris. 12, 87 p.

BALDNER L., 1888. L'histoire naturelle des eaux strasbourgeoises, suivies de notes zoologiques diverses par Ferdinand REIBER, Membre de la Société d'Histoire Naturelle de Colmar, 1888. Chez J. Noirier, Librairie, Rues des Serruriers, 27.

BUFFAULT P., 1925. Une écrevisse américaine acclimatée en France ; le Cambarus du Cher. Revue des Eaux et Forêts, $63: 274$.

CHANGEUX T., 1996. Premiers résultats de l'enquête express écrevisses. Conseil Supérieur de la Pêche, Circulaire D0003 du 15/01/1995, 5 p. 
CHANGEUX T., 2003. Evolution de la répartition des écrevisses en France métropolitaine selon les enquêtes nationales réalisées par le Conseil Supérieur de la Pêche de 1977 à 2001. Bull. Fr. Pêche Piscic., (CRAYNET, Volume 1) 370-371 : 17-41.

COLLAS M., 1995. Distribution de deux espèces d'écrevisses dans la partie mosellane de la Réserve de Biosphère des Vosges du Nord. Annales Scientifiques de la Réserve de la Biosphère des Vosges du Nord. T.4 : 55-67.

COLLAS M., 1996. Distribution de quatre espèces d'écrevisses dans le département de la Moselle. Fédération des AAPPMA de la Moselle. Document Ronéotypé. 34 p.+Annexes.

COLLAS M., SALEK X., mars 2002. Description d'un cas de peste ou Aphanomycose dans le département des Vosges. L'astaciculteur de France -70, p2-6.

COLLAS M., HORNIER E., HIESIGER P., STEIN A., EBERHARDT J., MULLER P., SCHWAB F., 2005a. L'écrevisse des torrents (Austropotamobius torrentium), Suivi de la population du Bremmelbach (Bas-Rhin) et du Gailbach (Moselle). L'Astaciculteur de France : 88, 6-25.

COLLAS M., MILLARD R., FROMAGET V., LAFON S., 2005b. Enquête sur le transport et la commercialisation des écrevisses du Léman. Conseil Supérieur de la Pêche, Délégation Régionale de Metz, 12 p.+ annexes.

COLLAS M., MILLARD R., HAZEMANN C., 2006. Les écrevisses en France - Ecrevisses exotiques : la menace...Conseil Supérieur de la Pêche, Fédération des AAPPMA des Vosges, plaquette comprenant un poster et $4 \mathrm{p}$.

CUKERZIS J., 1977. Interspecific competition in closely related species of crayfish (Astacus astacus, Astacus leptodactylus and Pacifastacus leniusculus, decapoda Crustacea). Dokl. Biol. Sci. ;229 (1/6) : 283-285. New York. [ISSN 0012-4996]

DAUDEY T., 2006. Diagnostic des peuplements astacicoles de la vallée du Dessoubre, Etudes des populations d'espèces invasives et autochtones et relation avec la qualité du milieu. Rapport de stage. Université de Franche-Comté, Fédération Départementale de Pêche et de Protection du Milieu Aquatique.

DE DROUIN DE BOUVILLE R., 1901. Le repeuplement des rivières en Meurthe-et-Moselle. Extrait des comptes-rendus des Sociétés Savantes en 1901, Sciences. Paris, Imprimerie Nationale, MDCCCCl.

DE DROUIN DE BOUVILLE R., 1906. Les repeuplements en écrevisses. Ed. Berger-Levrault \& Cie, Paris-Nancy, 113 p., avec 24 figures et 7 planches stéréographiques.

DUBOIS J.P, GILLET C., LAURENT P.J., MICHOUD M., 2003. Que sont devenues les populations d'écrevisses de la rive française du lac Léman - L'astaciculteur de France-Bulletin $\mathrm{n}^{\circ} 77$, p. 2-11.

DRUART D., MOSIMANN B., JULIEN C., 2007. Suivi de quatre années d'une population d'écrevisses du Pacifique (Pacifastacus leniusculus) sur un bassin abritant des écrevisses à pieds blancs (Austropotamobius pallipes) - Bassin du Saulon, Département de la Haute-Marne - Période 2003-2006. 24 p. et annexes.

FIERS V., GAUVRIT B., GAVAZZI E., HAFFNER P., MAURIN H. et coll., 1997. Statut de la faune de France métropolitaine. Statuts de protection, degrés de menace, statuts biologiques. Col. Patrimoines naturels, vol.24- Paris, Service du Patrimoine Naturel. IEGB/MNHN, Réserves naturelles de France, Ministère de l'Environnement, 225 p.

FRANCKHAUSER R., MACHINO Y., 2001. L'écrevisse de torrents (Austropotamobius torrentium) en France. L'Astaciculteur de France, 68, 2-5. 
HEFTI D. and STUCKI P., 2006. Crayfish management for Swiss waters. Bull. Fr. Pêche Piscic. 380-381: 937-950.

HORNIER E., COLLAS M., CLAUSS T., STORCK F., ANDRE S., HIESIGER P., 2002. L'écrevisse de torrent est toujours présente en Alsace - L'astaciculteur de France $\mathrm{N}^{\circ} 73$, p. 6-10

HOLDICH, D.M. et DOMANIESWSKI C.J., 1995. Studies on a mixed population of the crayfish Austropotamobius pallipes and Pacifastacus leniusculus in England, p. 3745. In M.C. GEDDES, DR FIELDER et A.M.M. RICHARDSON (eds), Freshwater crayfish, vol.10. Louisiana State University, Baton Rouge (Louisiana). [ISBN 0$9642382-2-5]$

HOLDICH D.M., READER J.P., ROGERS W.D. et HARLIOGLU M., 1995. Interaction between three species of crayfish (Austropotamobius pallipes, Astacus leptodactylus and Pacifastacus leniusculus). P. 46 - 56. In M.C. GEDDES, D.R. FIELDER et A.M.M. RICHARDSON (eds], Freshwater Crayfish, vol.10. Louisiana State University, Baton Rouge (Louisiana)). [ISBN 0-9642382 - 2 - 5]

HUET M., 1949. Aperçu des relations entre la pente et les populations piscicoles des eaux courantes. Schweiz. Z. Hydrol. II (3-4), p. 332-351.

JULIEN C., COLLAS M., GUIDOU F., 2005. Les écrevisses du département de la Marne, Synthèse de cinq années de prospections astacologiques, Période 2000-2004. Agence de l'Eau Seine-Normandie et Conseil Supérieur de la Pêche : 45 p.

KEITH P., ALLARDI J., MOUTOU B., 1992. Livre rouge des espèces menacées de poissons d'eau douce de France. Coll. Patrimoines Naturels, Vol. 10 S.F.F.- M.N.H.M., CSP, CEMAGREF, Min. Env., Paris, 111 p.

LAURENT P.J., 1983. Un siècle de transplantation d'écrevisses nord-américaines. C.R. Soc. Biogéogr. 59 (3c) : 394-404.

LAURENT P.J., 1987. Les enseignements de quarante années de statistiques douanières interprétées par un astacologue. Aqua Revue, $14: 15-19 ; 15: 35-39$.

LAURENT P.J., 1989. Les écrevisses dans le monde. L'Astaciculteur de France, 21 : 9-17.

LAURENT P.J., 1997. Introduction d'écrevisses en France et dans le monde, historique et conséquences. Bull. Fr. Pêche Piscic., Les introductions d'espèces dans les milieux aquatiques continentaux en métropole. Séminaire Ministère de l'Environnement, GIP HydrOsytème. №344-345 : 345-356.

LAURENT P.J., 2003. Astacus leptodactylus Escholtz, 1823, serait elle capable de stopper l'avance d'Orconectes limosus Rafinesque, 1817? L'astaciculteur de France 74 : 8-16.

LAURENT P.J., LELOIRN H., NEVEU A., 1991. Remarques sur l'acclimatation en France de Procambarus clarkii (Decapoda, Cambaridae). Bull. mens. Soc. Linn. Lyon, 60 (5) : 166-173.

LAURENT P.J., NICOLAS J. et PARIS L., 1997. Les repeuplements en Astacus astacus L. Synthèse des essais réalisés en Lorraine et en Morvan, enseignements à en tirer. L'Astaciculteur de France, N'51: $34-58$.

LAURENT P.J., SUSCILLON M., 1962. Les écrevisses en France. Annales Station Centrale Hydrobiologie Appliquée, 9 : 333-395 + 2pl.

LEGER L., 1924. Une nouvelle écrevisse dans les eaux françaises, notre de M.L. LEGER, présentée par M. Ch. GRAVIER. Comptes rendus de l'Académie des Sciences, T. 179: 1205-1207. 
LERAT D., PARIS L., BARAN P., 2006. Statut de l'écrevisse à pattes blanches (Austropotamobius pallipes Lereboullet, 1858) en Bourgogne : Bilan de 5 années de prospection. Bull. Fr. Pêche Piscic., 380-381: 867-882.

LEREBOULLET A., 1858. Description de deux nouvelles espèces d'écrevisses de nos rivières. Mem. Soc. Sci. Nat. Strasbourg, 5 (1) : 11 p. + 3 pl. (ISSN 1155-1127).

MACHINO Y., 1995. Austropotamobius torrentium (Schrank, 1803) existe bel et bien en France. L'Astaciculteur de France, 45, 17-19.

MACHINO Y., 1996. Nouveau site de l'écrevisse des torrents (Austropotamobius torrentium Schrank, 1803) en France. L'astaciculteur de France, 48, 2-3.

NEVEU A., 1998a. Présence de l'aphanomycose en France : suivi d'un foyer dans l'ouest de 1990 à 1998. L'astaciculteur de France, 57 : 2-6.

NEVEU A., 1998b. Pacifastacus leniusculus son rôle de vecteur et de réservoir de la peste des écrevisses (aphanomycose). Etat actuel des connaissances. L'astaciculteur de France $N^{\circ} 57$, : 6-11.

NEVEU A., 2006. Les écrevisses étrangères sont-elles invasives ? Quelles caractéristiques expliquent leur développement ? L'astaciculteur de France, $N^{\circ} 86: 2-11$.

NEVEU A., BACHELIER E., 2003. Mortalités d'Austropotamobius pallipes sur le bassin de la Sèvre Niortaise, Présence de l'Aphanomycose. L'astaciculteur de France, 73, 2-4.

PEUPION A., 1898. Traité de pisciculture. Berger-Levrault \& Cie : Paris-Nancy, 657 p.

SOUTY-GROSSET C., HOLDICH D.M., NOËL P.Y., REYNOLDS J.D. et HAFFNER P. (eds), 2006. 6 Atlas of Crayfish in Europe. Museum national d'Histoire naturelle, Paris, 187 p. (Patrimoines naturels, 64).

STUCKI P. et ZAUG B., 2006. Plan d'action national pour les écrevisses. Office fédéral de l'environnement, $41 \mathrm{p}$.

TELEOS, Fédération de Pêche 39, Brigade CSP 39, 2004. Contribution à la recherche des causes de régression de l'écrevisse pieds blancs (Austropotamobius pallipes)Expérimentation dans le département du Jura de 2000 à 2003 - Recherche des causes insidieuses d'extinction, Caractérisation des exigences habitationnelles minimales, Mise au point d'un système expert. Programme commandité et coordonné par le Conseil Supérieur de la Pêche, 99 p.

VERNEAUX J., 1973. Cours d'eau de Franche Comté. Recherches écologiques sur le réseau hydrographique du Doubs -Essai de biotypologie. Mém. Thèse Doct. D'Etat, Univ. Besançon, 260 p.

VIGNEUX D., 1980. Enquête sur les écrevisses en France. Dépouillement et compterendu. Rapport Conseil Supérieur de la Pêche, 156 p.

VIGNEUX E., KEITH P., NOËL P., (edit.), 1993. Atlas préliminaire des Crustacés Décapodes d'eau douce de France. Coll. Patrimoines Naturels, Vol.14, S.F.F., B.I.M.M.M.N.H.N., C.S.P., Min. Env., Paris, 55 p.

TAYLOR C.A., 2000. Systematic studies of the Orconectes juvenilis Complex (Decapoda: Cambaridae) with descriptions of two new species - Journal of Crustacean Biology 20 (1) / 132-152.

VOGT G., 1999. Diseases of European freshwater crayfish, with particular emphasis on interspecific transmission of pathogens. In GHERARDI F. \& HOLDICH D.M., Crayfish in Europe as alien species, 87-106. 


\section{Annexe 1}

Questionnaire utilisé dans l'enquête express écrevisses 2006

\section{Annex 1}

Questionnaire form used in the 2006 crayfish survey

\section{NOTES EXPLICATIVES POUR L'ENQUÊTE EXPRESS ECREVISSE}

Pour alléger le questionnaire les informations à fournir concernent le département dans son ensemble

\section{(1) Code espèce}

ASA : Astacus astacus, écrevisse à pattes ou à pieds rouges

APP : Austropotamobius pallipes, écrevisse à pieds blancs

APT : Austropotamobius torrentium, écrevisse des torrents

ASL : Astacus leptodactylus, écrevisse à pattes grêles ou turque

$\mathrm{OCL}$ : Orconectes limosus, écrevisse américaine

PFL : Pacifastacus leniusculus, écrevisse de Californie

PCC : Procambarus clarkii, écrevisse de Louisiane

Autres espèces d'écrevisses à préciser par un code indiqué dans " observations générales ":

(2) Tendance majeure dans le département depuis l'inventaire de 2001

-- : Disparition de populations

- : Maintien des populations avec diminution des effectifs

0 : Maintien des populations et des effectifs

+ : Maintien des populations avec augmentation des effectifs

++ : Apparition de populations

NSP : Ne sais pas

(3) Effort de prospection depuis la dernière enquête (été 2001)

0 : Aucune prospection pour l'espèce

+ : Prospection faible pour l'espèce (moins de $10 \mathrm{~h} . j \mathrm{j}$ par an)

++ : Prospection moyenne pour l'espèce (10-30 h.j par an)

+++ : Prospection importante pour l'espèce (plus de 30 h.j par an)

Remarque : une même journée peut concerner plusieurs espèces différentes.

(4) Indice d'abondance dans le département

0 : Absente dans les cours d'eau de la catégorie concernée

+ : Présence faible (comprise entre $1 \%$ et $30 \%$ du linéaire)

++ : Présence moyenne (comprise entre $30 \%$ et $60 \%$ du linéaire)

+++ : Présence importante (comprise entre $60 \%$ et $100 \%$ du linéaire)

NSP : Ne sais pas

(5) Nbre de sites :

Préciser pour chaque espèce le nombre de sites connus 


\section{(6) Exploitation par pêche dans le département}

I : Pêche interdite

0 : Pas d'exploitation (sans intérêt halieutique)

+ : Exploitation faible (capture accessoire)

++ : Exploitation moyenne (espèce cible)

+++ : Exploitation importante (espèce très recherchée)

NSP : Ne sais pas

Remarque : Les productions peuvent être faibles malgré une exploitation importante si les stocks sont petits.

\section{(7) Mortalité : une ligne par mortalité constatée}

«Espèce suspecte » à l'origine de la mortalité si connue

«Date » du premier constat depuis septembre 2001 («jour/mois/année » si constat CSP, « mois/ année » sinon)

"Origine »: inconnue, peste diagnostiquée, peste suspectée, thelohaniose diagnostiquée, thelohaniose suspectée, pollution diagnostiquée, pollution suspectée, autre (préciser) : 


\section{FICHE D'ENQUETE ECREVISSE}

\section{à renvoyer avant le 15 septembre 2006}

à

$$
\text { DR1 - DR2 - DR3 - DR4 - DR5 - DR6 - DR7 - DR8 - DR9 }
$$

Sur l'ensemble du département de :.

\begin{tabular}{|c|c|c|c|c|c|}
\hline $\begin{array}{c}\text { Espèce } \\
(1)\end{array}$ & $\begin{array}{c}\text { Présence } \\
\text { (oui/non) }\end{array}$ & $\begin{array}{c}\text { Tendance } \\
\text { majeure (2) }\end{array}$ & $\begin{array}{c}\text { Effort (3) } \\
\text { prospection }\end{array}$ & \multicolumn{2}{c|}{$\begin{array}{c}\text { Pêche :date../../.. } \\
\text { Ouverture - Fermeture }\end{array}$} \\
\hline ASA & & & & & \\
\hline APP & & & & & \\
\hline ASL & & & & & \\
\hline OCL & & & & & \\
\hline PFL & & & & & \\
\hline PCC & & & & & \\
\hline APT & & & & & \\
\hline
\end{tabular}

Cours d'eau 1 ère catégorie

\begin{tabular}{|c|c|c|c|}
\hline $\begin{array}{c}\text { Espèce } \\
(1)\end{array}$ & $\begin{array}{c}\text { Ind.(4) } \\
\text { d'abon. }\end{array}$ & $\begin{array}{c}\text { Nb de } \\
\text { sites } \\
(5)\end{array}$ & $\begin{array}{c}\text { Pêche } \\
\text { Amateur } \\
(6)\end{array}$ \\
\hline ASA & & & \\
\hline APP & & & \\
\hline ASL & & & \\
\hline OCL & & & \\
\hline PFL & & & \\
\hline PCC & & & \\
\hline APT & & & \\
\hline & & & \\
\hline
\end{tabular}

* Consulter les notes explicatives jointes pour remplir les tableaux
Cours d'eau de $2^{\text {nde }}$ catégorie

\begin{tabular}{|c|c|c|c|c|}
\hline $\begin{array}{c}\text { Espèce } \\
(1)\end{array}$ & $\begin{array}{c}\text { Ind.(4) } \\
\text { d'abon. }\end{array}$ & $\begin{array}{c}\text { Nb de } \\
\text { sites } \\
(5)\end{array}$ & $\begin{array}{c}\text { Pêche } \\
\text { amateur } \\
\text { (6) }\end{array}$ & $\begin{array}{c}\text { Pêche } \\
\text { pro. } \\
(6)\end{array}$ \\
\hline ASA & & & & \\
\hline APP & & & & \\
\hline ASL & & & & \\
\hline OCL & & & & \\
\hline PFL & & & & \\
\hline PCC & & & & \\
\hline APT & & & & \\
\hline & & & & \\
\hline
\end{tabular}

T.S.V.P 
Plans d'eau publics

(domaniaux ou communaux)

\begin{tabular}{|c|c|c|c|}
\hline $\begin{array}{c}\text { Espèce } \\
(1)\end{array}$ & $\begin{array}{c}\mathrm{Nb} \text { de } \\
\text { sites }\end{array}$ & $\begin{array}{c}\text { Pêche(6) } \\
\text { amateur }\end{array}$ & $\begin{array}{c}\text { Pêche(6) } \\
\text { pro. }\end{array}$ \\
\hline ASA & & & \\
\hline APP & & & \\
\hline ASL & & & \\
\hline OCL & & & \\
\hline PFL & & & \\
\hline PCC & & & \\
\hline APT & & & \\
\hline & & & \\
\hline
\end{tabular}

$\mathrm{Nb}$ total approximatif de plans d'eau publics dans le département :......
Plans d'eau privés ou unités d'exploitation

\begin{tabular}{|c|c|c|}
\hline $\begin{array}{c}\text { Espèce } \\
(1)\end{array}$ & $\begin{array}{c}\text { Nb de } \\
\text { sites }\end{array}$ & $\begin{array}{c}\text { Production (en } \mathrm{kg} \text { ) } \\
\text { annuelle estimée }\end{array}$ \\
\hline ASA & & \\
\hline APP & & \\
\hline ASL & & \\
\hline OCL & & \\
\hline PFL & & \\
\hline PCC & & \\
\hline APT & & \\
\hline & & \\
\hline
\end{tabular}

$\mathrm{Nb}$ total approximatif de plans d'eau privés ou d'unités d'exploitation dans le département :......

\section{Mortalités (7)}

\begin{tabular}{|l|l|l|l|}
\hline $\begin{array}{c}\text { Espèce } \\
\text { victime (1) }\end{array}$ & $\begin{array}{c}\text { Espèce } \\
\text { suspecte (1) }\end{array}$ & Date & Origine \\
\hline & & & \\
\hline & & & \\
\hline & & & \\
\hline & & & \\
\hline & & & \\
\hline & & & \\
\hline & & & \\
\hline & & & \\
\hline
\end{tabular}

\section{OBSERVATIONS GENERALES}

